\title{
THE ACS SURVEY OF GALACTIC GLOBULAR CLUSTERS. VII.* RELATIVE AGES
}

\author{
Antonio Marín-Franch ${ }^{1,2}$, Antonio Aparicio ${ }^{3}$, Giampaolo Piotto $^{4}$, Alfred Rosenberg ${ }^{1}$, Brian Chaboyer $^{5}$, \\ Ata Sarajedini ${ }^{2}$, Michael Siegel ${ }^{6}, \mathrm{JAy}_{\text {Anderson }}{ }^{7}$, Luigi R. Bedin ${ }^{7}$, AAron DotTer $^{5}$, MARen Hempel $^{2}$, Ivan King ${ }^{8}$, \\ Steven Majewski ${ }^{9}$, Antonino P. Milone ${ }^{4}$, Nathaniel Paust ${ }^{7}$, and I. Neill Reid ${ }^{7}$ \\ ${ }^{1}$ Instituto de Astrofísica de Canarias, Vía Láctea s/n, E-38200 La Laguna, Spain; amarin@iac.es, alf@iac.es \\ ${ }^{2}$ Department of Astronomy, University of Florida, 211 Bryant Space Science Center, Gainesville, FL 32611, USA; amarin@iac.es, ata@astro.ufl.edu, \\ hempel@astro.ufl.edu \\ ${ }^{3}$ University of La Laguna and Instituto de Astrofísica de Canarias, E-38200 La Laguna, Spain; antapaj@iac.es \\ ${ }^{4}$ Dipartimento di Astronomia, Università di Padova, 35122 Padova, Italy; giampaolo.piotto@unipd.it, antonino.milone@oapd.inaf.it \\ 5 Department of Physics and Astronomy, Dartmouth College, 6127 Wilder Laboratory, Hanover, NH 03755, USA; chaboyer@heather.dartmouth.edu, \\ Aaron.L.Dotter@dartmouth.edu \\ ${ }^{6}$ University of Texas, McDonald Observatory, 1 University Station, C1402, Austin, TX 78712, USA; siegel@astro.as.utexas.edu \\ ${ }^{7}$ Space Telescope Science Institute, 3700 San Martin Drive, Baltimore, MD 21218, USA; jaynder@stsci.edu, bedin@stsci.edu, paust@stsci.edu, inr@stsci.edu \\ 8 Department of Astronomy, Univ. of Washington, Box 351580, Seattle, WA 98195-1580, USA; king@astro.washington.edu \\ ${ }^{9}$ Department of Astronomy, University of Virginia, P.O. Box 400325, Charlottesville, VA 22904-4325, USA; srm4n@virginia.edu \\ Received 2008 July 14; accepted 2008 December 28; published 2009 March 25
}

\begin{abstract}
The ACS Survey of Galactic globular clusters is a Hubble Space Telescope Treasury program designed to provide a new large, deep, and homogeneous photometric database. Based on observations from this program, we have measured precise relative ages for a sample of 64 Galactic globular clusters by comparing the relative position of the clusters' main-sequence (MS) turnoffs, using MS fitting to cross-compare clusters within the sample. This method provides relative ages to a formal precision of $2 \%-7 \%$. We demonstrate that the calculated relative ages are independent of the choice of theoretical model. We find that the Galactic globular cluster sample can be divided into two groups-a population of old clusters with an age dispersion of $\sim 5 \%$ and no age-metallicity relation, and a group of younger clusters with an age-metallicity relation similar to that of the globular clusters associated with the Sagittarius dwarf galaxy. These results are consistent with the Milky Way halo having formed in two phases or processes. The first one would be compatible with a rapid $(<0.8 \mathrm{Gyr})$ assembling process of the halo, in which the clusters in the old group were formed. Also these clusters could have been formed before re-ionization in dwarf galaxies that would later merge to build the Milky Way halo as predicted by $\Lambda$ CDM cosmology. However, the galactocentric metallicity gradient shown by these clusters seems difficult to reconcile with the latter. As for the younger clusters, it is very tempting to argue that their origin is related to their formation within Milky Way satellite galaxies that were later accreted, but the origin of the age-metallicity relation remains unclear.
\end{abstract}

Key words: Galaxy: evolution - Galaxy: formation - globular clusters: general

\section{INTRODUCTION}

One of the keys to understanding the structure and evolution of the Milky Way is the ability to divide its stars and clusters into separate Galactic populations as first hinted at by Oort (1926) and formally introduced by Baade (1944). In the early 1950s, Sandage published the first deep color-magnitude diagrams (CMDs) of the northern Galactic globular clusters (GGCs) M3 and M92 (Sandage 1953; Arp et al. 1953) and discovered the faint sequence of stars similar to the main sequence (MS) of Population I. It was almost immediately realized that GGCs are ancient compared to the stars in the Solar neighborhood.

Over the last half century, it has become clear that GGCs are astronomical fossils providing important information on the formation and evolution of the Milky Way. One characteristic of GGCs that makes them so valuable is that they are the oldest Galactic objects for which reliable ages can be measured.

GGC ages are of great interest to a variety of cosmological and comogonical issues since their absolute ages place a lower limit on the age of the Universe. Unfortunately, absolute age

\footnotetext{
* Based on observations with the NASA/ESA Hubble Space Telescope, obtained at the Space Telescope Science Institute, which is operated by AURA, Inc., under NASA contract NAS 5-26555, under program GO-10775 (PI: A. Sarajedini).
}

measurements are still compromised by a number of uncertainties, particularly GGC distances and foreground reddenings as well as metallicities, bolometric corrections, and some poorly known aspects of the stellar evolution. Relative ages are almost (although not totally) free of these effects and can still reveal fundamental information about Galaxy formation mechanisms and timescales. For this reason, they have become of paramount importance in the context of the study of GGC.

The study of GGC ages has evolved apace with improvements in instrumentation, in our theoretical understanding of stellar evolution and with the advance of computational facilities. Reliable age determination requires deep photometry that reaches at least the main-sequence turnoff (MSTO), together with a good understanding of the stellar evolution theoretical modeling. During the first half of the $20^{\text {th }}$ century, the available instrumentation did not allow observations deeper than the horizontal branch of most GGCs. Over the past three decades, however, low-quantum efficiency nonlinear photographic imaging has given way to digital imaging using high-quantum efficiency linear CCDs. This has resulted in ever deeper and more precise cluster CMDs that reached the MSTO and allowed the application of age dating techniques. First relative age studies were carried out by Gratton (1985), Peterson (1987), Sarajedini \& King (1989) and Sarajedini \& Demarque (1990), as representative examples. However, uncertainties in both the input physics 
(Chaboyer et al. 1998) and the color- $T_{\text {eff }}$ transformation of the theoretical models into the observational plane (Buonanno et al. 1998) constitute a significant source of errors in the final relative age determination, particularly when ages of clusters with different metallicities are compared. In the last decade, many efforts have been made in GGC relative ages, improving our understanding with each technological or theoretical advance (e.g., Chaboyer et al. 1996; Richer et al. 1996; Stetson et al. 1996; Salaris \& Weiss 1998; Buonanno et al. 1998; Rosenberg et al. 1999; VandenBerg 2000; De Angeli et al. 2005).

Relative ages can be estimated to high precision by measuring the position of the MSTO relative to CMD features that have little or no age dependence (Stetson et al. 1996; Sarajedini et al. 1997). However, a key aspect of any relative age experiment is having a large homogenous database of globular cluster photometry. Such databases have only recently appeared in the literature but have significantly advanced our understanding of Milky Way formation and evolution. The largest dedicated efforts are those of Rosenberg et al. (2000a, 2000b), a groundbased survey of 35 nearby GGCs (Rosenberg et al. 1999), and De Angeli et al. (2005), an analysis of 55 clusters from Piotto et al. (2002)'s Hubble Space Telescope (HST) snapshot catalog.

These and other recent relative age studies (Buonanno et al. 1998; Salaris \& Weiss 1998; Rosenberg et al. 1999; VandenBerg 2000; De Angeli et al. 2005) consistently point toward a scenario in which metal-poor clusters are largely coeval but metal-rich clusters show some age dispersion, $\sim 1.0 \mathrm{Gyr}$ (rms), and a possible age-metallicity relation. No correlation between age and Galactocentric distance has been conclusively demonstrated.

In this paper, we present an analysis of a uniform set of HST/ACS data for 64 GGCs. ${ }^{10}$ This data set, previously introduced by Sarajedini et al. (2007), has produced well-defined CMDs for all of the target clusters from the tip of the red giant branch (RGB) to at least $\sim 6.5 \mathrm{mag}$ below the MSTO (Anderson et al. 2008). The determination of GGC ages was a principle goal of the HST/ACS survey and exposures times were defined to maximize the signal-to-noise ratio $(\mathrm{S} / \mathrm{N})$ at the MSTO. This makes the survey an excellent resource for the evaluation of relative ages.

Due to the difficulty in determining the position of the $\mathrm{MSTO}^{11}$ to the high precision required for age studies, many different methods have been developed in previous relative ages works (see Meissner \& Weiss 2006 for a complete discussion). The two most used relative age indicators are those based on the color difference between the MSTO and the RGB at a given magnitude level (horizontal method), and the magnitude difference between the MSTO and the zero-age $\mathrm{HB}^{12}$ (vertical method). VandenBerg et al. (1990) were pioneers in measuring GGC relative ages making use of the horizontal method. They essentially derived relative ages comparing the color difference between the MSTO and the location of a well-defined point in the lower $\mathrm{RGB}$, located $2.5 \mathrm{mag}$ brighter than a point in the upper MS that is 0.05 mag redder than the MSTO. However, horizontal method results are sensitive to the assumed value of the mixing length parameter and to the color- $T_{\text {eff }}$ transformation of the theoretical

\footnotetext{
10 Sarajedini et al. (2007) presented observations of 65 GCs, but Pal 2 has been left out of this study due to its high-differential reddening.

11 The MSTO point is the current position in the CMD where stars in the cluster start burning hydrogen in a shell and are leaving the MS. The empirical MSTO is then the bluest point on the MS-to-subgiant-branch portion of the cluster's CMD.

12 The zero-age HB is the position in the CMD where stars in the cluster start burning helium into carbon and oxygen in a helium-rich core and hydrogen into helium in a shell.
}

models into the observational plane. As a result, relative ages determined using the horizontal method are model dependent. Potentially, more reliable age indicators are those related to the brightness of the MSTO, in particular the vertical method. First surveys of GGC relative ages using the vertical method were carried out by Gratton (1985) and Peterson (1987). They measured relative ages from a sample of 26 and 41 GGCs, respectively, collected from the available literature. Later on, Sarajedini \& King (1989) used the brightness difference between the HB and the MSTO to estimate the ages of 31 GGCs. Nevertheless, accurate determination of the zero-age HB is delicate, in particular for the clusters at the extreme boundaries of the metallicity distribution, which generally have a red (or blue) $\mathrm{HB}$ and no RR Lyrae stars. Moreover, the dependence of the zero-age HB luminosity on the cluster metallicity is still controversial.

In this paper, we use an alternative approach. Our data reach at least $\sim 6.5$ mag below the MSTO, providing a very welldefined MS. This allows, for the first time, the use of relative MS fitting for a large number of clusters. As will be shown later, the relative MS-fitting procedure used in this study does essentially the following. The MS of two clusters, with similar metallicities, is superimposed in the CMD by shifting one of the clusters' mean ridge line in both color and magnitude (explained in detail in Section 4). This way, any differences between the two clusters' distance and reddening are compensated, and an intrinsic MSTO magnitude difference is obtained. This relative MSTO brightness is then used to derive relative ages. This allows us to use what is, in essence, an improved version of the traditional vertical method that substitutes the well-defined MS for the contentious zero-age HB.

We also take the additional step of comparing our observational measures to updated theoretical models. We have primarily used the most recent state-of-art models of Dotter et al. (2007) (hereafter D07), transformed to the observational plane with an empirical color- $T_{\text {eff }}$ transformation. However, we also compare our results to the models of Pietrinferni et al. (2004) (hereafter P04), Bertelli et al. (1994) (hereafter B94), and Girardi et al. (2000) (hereafter G00). We find that the various theoretical models agree closely on the MSTO absolute magnitude and color, resulting in negligible model dependence.

This paper is organized as follows. Section 2 describes the observations and data reduction pipeline, and Section 3 presents the globular cluster database. The mean ridge line fitting and MS-fitting procedures used in this study are described in Section 4. The analysis of the uncertainties is presented in Section 5. Finally, relative age results and a discussion are presented in Sections 6 and 7, respectively. The paper ends with a summary of conclusions (Section 8).

\section{OBSERVATIONS AND DATA REDUCTION}

Observations were performed with the ACS/WFC instrument on board the HST. A total of 65 GGCs were observed in the $F 606 W(\sim V)$ and $F 814 W(\sim I)$ filters. Due to its highdifferential reddening, Pal 2 is not considered here.

Details on the observations, data reduction, and photometry are given in Sarajedini et al. (2007) and Anderson et al. (2008). For most globular clusters, the observation and data reduction pipeline produce $\sim 12$ mag of precise photometry, extending from nearly the tip of the RGB to several magnitudes below the MSTO. In general, the photometry reaches a depth corresponding to an approximate stellar mass of $\sim 0.2 M_{\odot}$.

This uniform and deep photometry offers a database of unprecedented quality, opening a window for new data analysis 
approaches. In particular, cluster MSs are so well defined that it is possible, for the first time, to apply relative MS-fitting techniques to a large number of clusters within the same database.

\section{THE DATABASE}

The position of the MSTO depends not only upon age but also upon metallicity. This effect requires the use of a large and homogeneous metallicity database in order to determine accurate relative ages. To account for this, we have adopted the metallicities listed in the Rutledge et al. (1997) catalog ${ }^{13}$ over both the Carretta \& Gratton (1997) (hereafter CG) and the Zinn \& West (1984) (hereafter ZW) metallicity scales. This catalog is maybe not as precise as a true compilation of metallicities based on high-dispersion spectra, but it has the advantage of being homogeneous. For clusters not listed in Rutledge et al. (1997), the original metallicity listed in the ZW catalog has been transformed to the CG metallicity scale using the following equation (Carretta et al. 2001):

$$
\begin{aligned}
{[\mathrm{Fe} / \mathrm{H}]_{\mathrm{CG}}=0.61 } & +3.04[\mathrm{Fe} / \mathrm{H}]_{\mathrm{ZW}}+1.981[\mathrm{Fe} / \mathrm{H}]_{\mathrm{ZW}}^{2} \\
& +0.532[\mathrm{Fe} / \mathrm{H}]_{\mathrm{ZW}}^{3} .
\end{aligned}
$$

Lyngå 7, E3, and Pal 1 metallicities are not listed in the previous catalogs. Lyngå7 and E3 metallicities in the CG scale have been calculated from the ZW metallicities of Tavarez \& Friel (1995) and Harris (1996), respectively. For Pal 1, we used the measure of Rosenberg et al. (1998).

Table 1 presents the list of our target GGCs, grouped by metallicity. Column 3 gives the cluster identity while Columns 4 and 5 present the adopted metallicities in the $\mathrm{ZW}$ and $\mathrm{CG}$ scales, respectively. Column 6 lists the Galactocentric distance $\left(r_{\mathrm{GC}}\right.$, in kpc), taken from Harris (1996).

\section{MEAN RIDGE LINES AND MS-FITTING METHOD}

In this section, we describe the general properties of the cluster mean ridge line fitting procedure. We then use the derived ridge lines to perform relative MS fitting between clusters.

\subsection{Mean Ridge Lines and MSTO Apparent Magnitude}

In order to determine accurate relative ages for each cluster, a precise determination of the MS and RGB mean ridge line is required. With this aim, we have created a new software package for the ACS program. It is worth mentioning that mean ridge lines are determined in the observational plane $(F 606 \mathrm{~W}-$ $F 814 W, F 814 W)$ instead of $(F 606 W-F 814 W, F 606 W)$, as the SGB appears more vertical in the CMD and the following procedure produces more accurate results. For the rest of the analysis, the $(F 606 W-F 814 W, F 606 W)$ plane is used.

For each globular cluster, the mean ridge line is determined in two steps (Figure 1). First, color histograms are computed for stars grouped in $0.4 \mathrm{mag}(F 814 \mathrm{~W})$ wide bins. The value of 0.4 mag was determined experimentally as a compromise between two effects - the low resolution produced by larger bins and the noisy ridge line produced by smaller bins. Color histograms are constructed along the RGB and MS with a step of 0.04 mag (a tenth of the bin size) in F814W. That is, a moving bin of fixed width $(0.4 \mathrm{mag})$, with successive steps of $0.04 \mathrm{mag}$, is used. A preliminary mean ridge line is then derived with

\footnotetext{
13 Rutledge et al. (1997) presented a large homogeneous metallicity database
} (over 71 GGCs) based on calcium index (Ca II Triplet) measurements.
$(F 606 W-F 814 W)$ colors set at each histogram's maximum, and the $F 814 \mathrm{~W}$ magnitude of the bin center.

Once the preliminary mean ridge line has been determined, the fitting process is iterated three more times. However, we now use rotated histograms. For each $F 814 \mathrm{~W}$ value, the tangential angle of the mean ridge line is determined. This angle is used to rotate the reference system on which the histogram is computed. The $X$-axis of the new reference system is perpendicular to the preliminary mean ridge line at the considered $F 814 \mathrm{~W}$ magnitude. The histogram is then computed perpendicular to the preliminary mean ridge line and considering stars in the new reference system's $Y$-axis interval $[-0.04,0.04]$. The histogram's maximum is determined, defining a point of the new mean ridge line in the rotated reference system, $(\max , 0)$. Note that this point shows the correction to be applied to the preliminary mean ridge line. The coordinates of this point are de-rotated, and its location in the original reference system, the CMD, is calculated. The process is repeated for all the $F 814 \mathrm{~W}$ values to derive the revised mean ridge line, which is then smoothed to derive the final mean ridge line. Figure 2 shows some examples of mean ridge line fitting results. Four selected clusters covering the metallicity range are shown.

For clusters with multiple stellar populations, such as NGC 1851 (Milone et al. 2008), NGC 2808 (Piotto et al. 2007), M54 (Siegel et al. 2007) and $\omega$ Cen (Lee et al. 1999; Pancino et al. 2000; Bedin et al. 2004), the mean ridge line fitting procedure fits the dominant population (see Figure 1).

The empirical MSTO is the bluest point on the mean ridge line. It is worth mentioning that the final mean ridge line consists of a discrete number of points, it is not a continuous line. For this reason, in order to determine the MSTO position, a continuous spline has been fitted to the MS-to-subgiant-branch portion of the final mean ridge line. Its bluest point is then adopted as the empirical MSTO. As an example, the position of the MSTO is marked with an open circle in Figure 2. In order to increase the precision of the MSTO position, the mean ridge lines have been determined 10 times for each cluster, with the starting point of the histograms offset in steps of 0.004 mag. The MSTO color and apparent magnitude have been determined as the mean of the 10 different measurements.

This method provides a consistently defined mean ridge line, minimizing the effects of the binary population and differential reddening (see Section 5). Additionally, the rotated coordinate system ensures that the best fit is derived around the steeply curved MSTO and subgiant branch region of the CMD. For high-metallicity clusters, in particular, the subgiant branch is nearly horizontal and the more commonly used mean ridge line determination method (drawing an approximate ridge line, rendering the sequence to a nearly vertical line and then taking histograms in small ranges of magnitude) lacks the resolution around the subgiant branch needed for precise age measurement.

\subsection{MS-Fitting Method}

The CMD location of the faint MS of a GGC is independent of its age, but highly dependent upon its metallicity. For this reason, we have divided the HST/ACS sample into six metallicity groups (see Table 1 ) using the following divisions: $[\mathrm{Fe} / \mathrm{H}]$ $=-0.3,-0.8,-1.1,-1.3,-1.5,-1.8$, and -2.3 . For each group, a reference cluster has been chosen: NGC 6304, NGC 6723, NGC 6981, NGC 6681, NGC 6101, and NGC 4590, respectively. Reference clusters have been selected to have lowdifferential reddening, low field star contamination, and a welldefined MS. Using the derived mean ridge lines, we performed 
Table 1

Globular Cluster Parameters

\begin{tabular}{|c|c|c|c|c|c|}
\hline Ref. Cluster & Metallicity Group & Name & {$[\mathrm{Fe} / \mathrm{H}]_{\mathrm{ZW}}$} & {$[\mathrm{Fe} / \mathrm{H}]_{\mathrm{CG}}$} & $r_{\mathrm{GC}}$ \\
\hline NGC 6304 & $-0.8 \leqslant[\mathrm{Fe} / \mathrm{H}]_{\mathrm{CG}}<-0.3$ & $\begin{array}{c}\text { NGC } 0104 \\
\text { NGC } 5927 \\
\text { NGC } 6304 \\
\text { NGC } 6352 \\
\text { NGC } 6366 \\
\text { NGC } 6388 \\
\text { NGC } 6441 \\
\text { NGC } 6496 \\
\text { NGC } 6624 \\
\text { NGC } 6637 \\
\text { NGC } 6838 \\
\text { LYNGÅ7 } \\
\text { PAL 1 } \\
\text { TERZAN7 }\end{array}$ & $\begin{array}{l}-0.71 \\
-0.32 \\
-0.38 \\
-0.50 \\
-0.58 \\
-0.74 \\
-0.59 \\
-0.50 \\
-0.50 \\
-0.72 \\
-0.58 \\
-0.62 \\
-0.60 \\
-0.05\end{array}$ & $\begin{array}{l}-0.78 \\
-0.64 \\
-0.66 \\
-0.70 \\
-0.73 \\
-0.77 \\
-0.60 \\
-0.70 \\
-0.70 \\
-0.78 \\
-0.73 \\
-0.64 \\
-0.70 \\
-0.56\end{array}$ & $\begin{array}{c}7.4 \\
4.5 \\
2.2 \\
3.3 \\
5.0 \\
3.2 \\
3.9 \\
4.3 \\
1.2 \\
1.9 \\
6.7 \\
4.2 \\
17.0 \\
16.0\end{array}$ \\
\hline NGC 6723 & $-1.1 \leqslant[\mathrm{Fe} / \mathrm{H}]_{\mathrm{CG}}<-0.8$ & $\begin{array}{c}\text { NGC } 0362 \\
\text { NGC } 1261 \\
\text { NGC } 1851 \\
\text { NGC } 6121 \\
\text { NGC } 6171 \\
\text { NGC } 6362 \\
\text { NGC } 6652 \\
\text { NGC } 6717 \\
\text { NGC } 6723 \\
\text { E3 } \\
\text { PAL } 12\end{array}$ & $\begin{array}{l}-1.33 \\
-1.32 \\
-1.23 \\
-1.27 \\
-1.09 \\
-1.18 \\
-0.99 \\
-1.33 \\
-1.12 \\
-0.80 \\
-0.82\end{array}$ & $\begin{array}{l}-1.09 \\
-1.08 \\
-1.03 \\
-1.05 \\
-0.95 \\
-0.99 \\
-0.97 \\
-1.09 \\
-0.96 \\
-0.83 \\
-0.83\end{array}$ & $\begin{array}{c}9.4 \\
18.2 \\
16.7 \\
5.9 \\
3.3 \\
5.1 \\
2.8 \\
2.4 \\
2.6 \\
7.6 \\
15.0\end{array}$ \\
\hline NGC 6981 & $-1.3 \leqslant[\mathrm{Fe} / \mathrm{H}]_{\mathrm{CG}}<-1.1$ & $\begin{array}{l}\text { NGC } 0288 \\
\text { NGC } 2808 \\
\text { NGC } 3201 \\
\text { NGC } 5904 \\
\text { NGC } 6218 \\
\text { NGC } 6254 \\
\text { NGC } 6584 \\
\text { NGC } 6715 \\
\text { NGC } 6752 \\
\text { NGC } 6981\end{array}$ & $\begin{array}{l}-1.40 \\
-1.36 \\
-1.53 \\
-1.38 \\
-1.40 \\
-1.55 \\
-1.51 \\
-1.54 \\
-1.54 \\
-1.50\end{array}$ & $\begin{array}{l}-1.14 \\
-1.11 \\
-1.24 \\
-1.12 \\
-1.14 \\
-1.25 \\
-1.30 \\
-1.25 \\
-1.24 \\
-1.21\end{array}$ & $\begin{array}{c}12.0 \\
11.1 \\
8.9 \\
6.2 \\
4.5 \\
4.6 \\
7.0 \\
19.2 \\
5.2 \\
12.9\end{array}$ \\
\hline NGC 6681 & $-1.5 \leqslant[\mathrm{Fe} / \mathrm{H}]_{\mathrm{CG}}<-1.3$ & $\begin{array}{c}\text { NGC } 4147 \\
\text { NGC } 5139 \\
\text { NGC } 5272 \\
\text { NGC } 5286 \\
\text { NGC } 5986 \\
\text { NGC } 6093 \\
\text { NGC } 6205 \\
\text { NGC } 6535 \\
\text { NGC } 6656 \\
\text { NGC } 6681 \\
\text { NGC } 6934 \\
\text { NGC } 7089 \\
\text { ARP } 2\end{array}$ & $\begin{array}{l}-1.77 \\
-1.59 \\
-1.66 \\
-1.70 \\
-1.65 \\
-1.75 \\
-1.63 \\
-1.78 \\
-1.75 \\
-1.64 \\
-1.54 \\
-1.61 \\
-1.74\end{array}$ & $\begin{array}{l}-1.50 \\
-1.35 \\
-1.34 \\
-1.41 \\
-1.35 \\
-1.47 \\
-1.33 \\
-1.51 \\
-1.49 \\
-1.35 \\
-1.32 \\
-1.31 \\
-1.45\end{array}$ & $\begin{array}{c}21.3 \\
6.4 \\
12.2 \\
8.4 \\
4.8 \\
3.8 \\
8.7 \\
3.9 \\
4.9 \\
2.1 \\
12.8 \\
10.4 \\
21.4\end{array}$ \\
\hline NGC 6101 & $-1.8 \leqslant[\mathrm{Fe} / \mathrm{H}]_{\mathrm{CG}}<-1.5$ & $\begin{array}{l}\text { NGC } 2298 \\
\text { NGC } 4833 \\
\text { NGC } 6101 \\
\text { NGC } 6144 \\
\text { NGC } 6397 \\
\text { NGC } 6541 \\
\text { NGC } 6809 \\
\text { TERZAN8 }\end{array}$ & $\begin{array}{l}-1.91 \\
-1.92 \\
-1.95 \\
-1.81 \\
-1.94 \\
-1.79 \\
-1.80 \\
-1.97\end{array}$ & $\begin{array}{l}-1.71 \\
-1.71 \\
-1.76 \\
-1.56 \\
-1.76 \\
-1.53 \\
-1.54 \\
-1.80\end{array}$ & $\begin{array}{c}15.7 \\
7.0 \\
11.1 \\
2.6 \\
6.0 \\
2.2 \\
3.9 \\
19.1\end{array}$ \\
\hline NGC 4590 & $-2.3 \leqslant[\mathrm{Fe} / \mathrm{H}]_{\mathrm{CG}}<-1.8$ & $\begin{array}{l}\text { NGC } 4590 \\
\text { NGC } 5024 \\
\text { NGC } 5053 \\
\text { NGC } 5466 \\
\text { NGC } 6341 \\
\text { NGC } 6779 \\
\text { NGC } 7078 \\
\text { NGC } 7099\end{array}$ & $\begin{array}{l}-2.11 \\
-2.04 \\
-2.10 \\
-2.22 \\
-2.24 \\
-2.20 \\
-2.13 \\
-2.05\end{array}$ & $\begin{array}{l}-2.00 \\
-1.86 \\
-1.98 \\
-2.20 \\
-2.16 \\
-2.00 \\
-2.02 \\
-1.92\end{array}$ & $\begin{array}{c}10.1 \\
18.3 \\
16.9 \\
16.2 \\
9.6 \\
9.7 \\
10.4 \\
7.1\end{array}$ \\
\hline
\end{tabular}




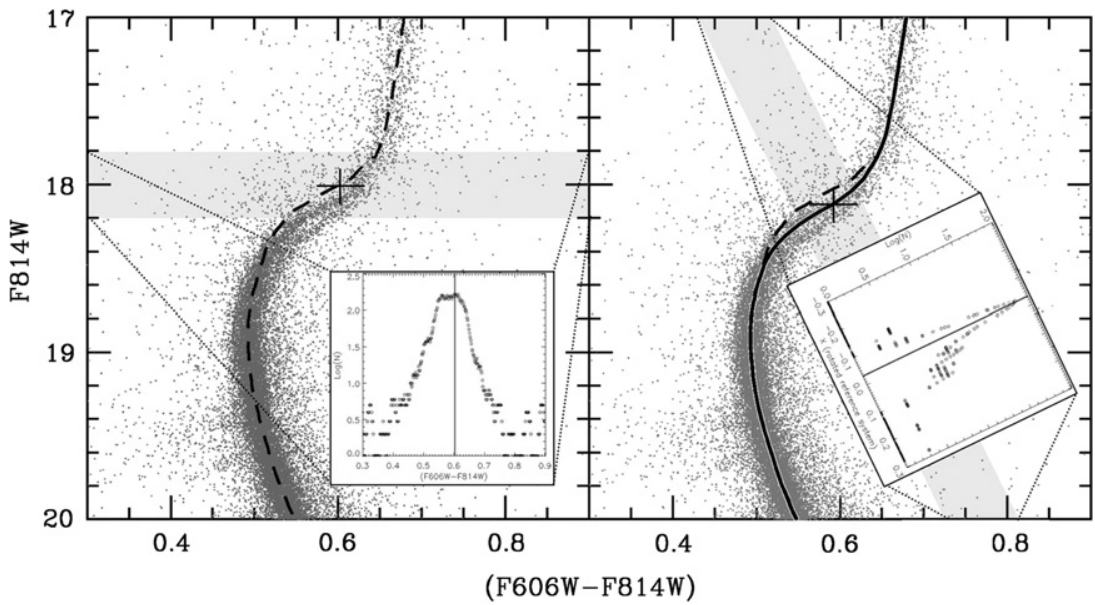

Figure 1. Mean ridge line fitting procedure using rotated histograms. This shows the analysis of NGC 1851. In short, the mean ridge line is determined in two steps. First, color histograms are computed for stars grouped in 0.4 mag $(F 814 W)$ wide bins (left panel). A preliminary mean ridge line is then derived with $(F 606 \mathrm{~W}-F 814 \mathrm{~W})$ colors set at each histogram's maximum, and the $F 814 \mathrm{~W}$ magnitude of the bin center. Second, we use rotated histograms, perpendicular to the preliminary mean ridge line, to derive the final one (right panel). See text for details.

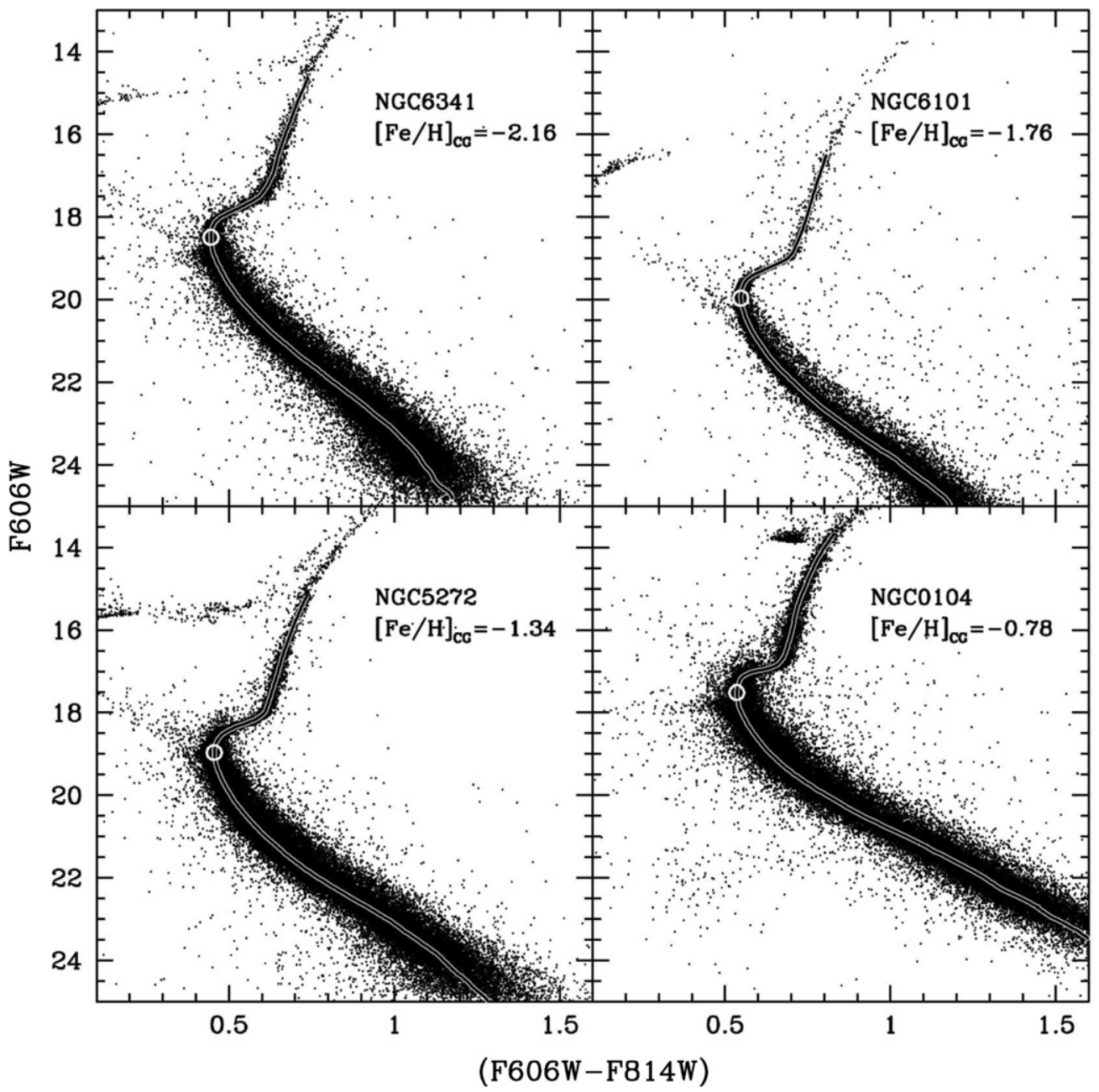

Figure 2. Examples of mean ridge line fitting in four selected clusters covering the metallicity range. The MSTO position is marked with an open circle.

MS and RGB fitting between each cluster in each metallicity group and the corresponding reference cluster. Figure 3 shows examples of the fitting, in which the mean ridge lines of clusters with $-1.3 \leqslant[\mathrm{Fe} / \mathrm{H}]_{\mathrm{CG}}<-1.1$ (solid lines) have been shifted in both magnitude and color to fit the reference cluster, NGC 6981 (dashed line). The fitting was performed in a least-squares fashion and taking into account two CMD regions that have little dependence upon cluster age. The two intervals are $\left[\left(M_{F 606 W}^{\mathrm{TO}}-2.5\right)<M_{F 606 W}<\left(M_{F 606 W}^{\mathrm{TO}}-1.5\right)\right]$ and $\left[\left(M_{F 606 W}^{\mathrm{TO}}+1.5\right)<M_{F 606 W}<\left(M_{F 606 W}^{\mathrm{TO}}+3.0\right)\right]$, with $M_{F 606 W}^{\mathrm{TO}}$ being the magnitude of the reference cluster's MSTO. These two intervals have been defined making use of D07 theoretical 

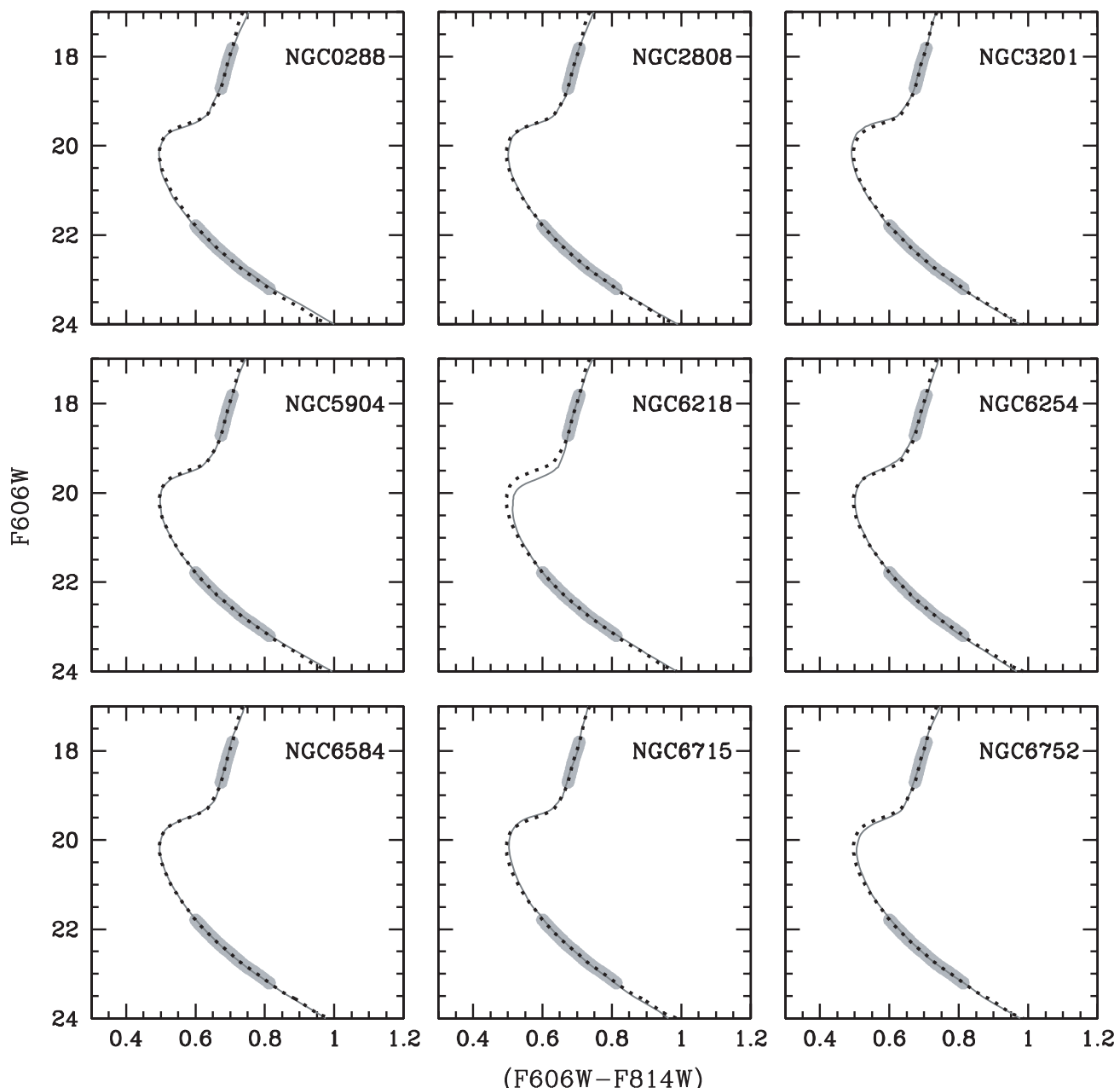

Figure 3. Examples of MS-fitting for the $-1.3 \leqslant[\mathrm{Fe} / \mathrm{H}]_{\mathrm{CG}}<-1.1$ metallicity group. The reference cluster is NGC 6981 (dashed line). Each cluster MRL (solid line) has been fitted to the reference cluster in the magnitude intervals $\left[\left(M_{F 606 W}^{\mathrm{TO}}-2.5\right)<M_{F 606 W}<\left(M_{F 606 W}^{\mathrm{TO}}-1.5\right)\right]$, and $\left[\left(M_{F 606 W}^{\mathrm{TO}}+1.5\right)<M_{F 606 W}<\left(M_{F 606 W}^{\mathrm{TO}}+3.0\right)\right]$ (shaded regions).

isochrones. In particular, D07 isochrones with similar metallicity and different ages were superimposed on the same CMD. Based on visual inspection, these two particular regions were found to have little dependence upon cluster age, and were adopted as the optimum intervals for the MS-fitting procedure. These regions are shaded in Figure 3. This fitting procedure produces unequivocal results, with no degeneracy between distance modulus and reddening.

The MS-fitting provides relative MSTO magnitudes for clusters inside each metallicity group. In order to calculate relative MSTOs between two consecutive metallicity groups, we applied the MS-fitting technique between the corresponding reference clusters. This was repeated for every two adjacent metallicity groups, producing a unified sample of relative MSTO magnitudes for all the analyzed clusters.

In order to compare relative MSTO measures to model predictions, we had to calculate the absolute magnitudes of the turnoffs. As the relative MSTO magnitudes are known, the MSTO absolute magnitude is only needed for one cluster. We chose the well-measured NGC 6752 cluster, adopting a subdwarf-based distance modulus of $(m-M)_{V}=13.24 \pm 0.08$ from Gratton et al. (2003). We calculate an MSTO magnitude of $M_{F 606 W}^{\mathrm{TO}}=4.02 \pm 0.08$ for NGC 6752 .

We performed similar calculations using the NGC 0104 and NGC 6397 distance moduli listed in Gratton et al. (2003) of
$(m-M)_{V}=13.50 \pm 0.08$ and $12.58 \pm 0.08$, respectively. We calculate MSTO absolute magnitudes of $M_{F 606 W}^{\mathrm{TO}}=4.03 \pm 0.08$ and $M_{F 606 W}^{\mathrm{TO}}=3.77 \pm 0.08$ for NGC 0104 and NGC 6397, respectively. As the relative MSTOs are known, NGC 6752's MSTO absolute magnitude can also be derived independently from NGC 0104 and NGC 6397 (3.72 \pm 0.08 and $3.86 \pm 0.08$, respectively). The mean of the three NGC 6752 MSTO absolute magnitude determinations is $M_{F 606 W}^{\mathrm{TO}}=3.87 \pm 0.15$, and we adopt this as the final MSTO zero point.

We note that any error in the MSTO magnitude zero point would affect absolute age determination, but not relative age measurements. For this reason, the zero point uncertainty is not taken into consideration during relative age determination.

Using the adopted zero point, we calculate MSTO absolute magnitudes for our entire sample, which are listed in Column 4 of Table 4. The listed uncertainties are determined in following section.

\section{MSTO UNCERTAINTIES}

Several effects must be taken into account when deriving the uncertainty associated with the measured MSTO magnitudes $\left(M_{F 606 W}^{\mathrm{TO}}\right)$. Among these, we address the cluster differential reddening, the binary fraction and total number of cluster stars. The total number of stars affects the MSTO magnitude 
Table 2

External Cross-check for the $\sigma_{\text {MRL }}^{2}$ Determination

\begin{tabular}{lcc}
\hline \hline Name & $\sigma_{\text {MRL }}$ & $\sigma_{\text {cross-check }}$ \\
\hline NGC 0104 & 0.011 & 0.011 \\
NGC 5024 & 0.024 & 0.013 \\
NGC 5272 & 0.011 & 0.012 \\
NGC 7089 & 0.018 & 0.006 \\
\hline
\end{tabular}

measurements in two ways. The first one is related to the total number of stars close to the MSTO that can be used to measure its magnitude: the smaller this number, the more uncertain the MSTO magnitude will be. On the other hand, a large number of cluster stars imply more severe crowding, and therefore less accurate magnitude measurement. To these uncertainties, we need to add the uncertainty from the MS-fitting and the mean ridge line determination procedure itself. The final uncertainty associated with the MSTO magnitude, $\sigma^{2}\left(M_{F 606 W}^{\mathrm{TO}}\right)$, is

$$
\sigma^{2}\left(M_{F 606 W}^{\mathrm{TO}}\right)=\sigma_{\mathrm{RED}}^{2}+\sigma_{\mathrm{BIN}}^{2}+\sigma_{\mathrm{NUM}}^{2}+\sigma_{\mathrm{CROW}}^{2}+\sigma_{\mathrm{MRL}}^{2}+\sigma_{\mathrm{MSF}}^{2},
$$

where $\sigma_{\mathrm{RED}}^{2}$ is the variance due to the differential reddening, $\sigma_{\mathrm{BIN}}^{2}$ due to the binary star population, $\sigma_{\mathrm{NUM}}^{2}$ due to the number of cluster stars, $\sigma_{\text {CROW }}^{2}$ due to the crowding, $\sigma_{\text {MRL }}^{2}$ is the MSTO's magnitude variance from the mean ridge line determination, and $\sigma_{\mathrm{MSF}}^{2}$ from the MS-fitting method. It is worth noting that field star contamination does not affect the MSTO determination. Our fields cover the central region in each cluster, and the ratio of cluster stars to field stars is very high. Moreover, as noted above, the mean-ridge-line-fitting method fits the dominant population, ignoring both field stars and blue stragglers.

As has been described in Section 4.1, each MSTO's magnitude has been determined as the mean of the MSTO's magnitude obtained from 10 different mean ridge line fittings. Here, we assume that the variance associated with the mean ridge line determination, $\sigma_{\mathrm{MRL}}^{2}$, is the variance of the previous 10 measurements. Typical values of $\sigma_{\mathrm{MRL}}$ are in the range $\sim 0.01-0.04$ mag.

With the aim of performing an external cross-check for the $\sigma_{\text {MRL }}^{2}$, the MSTO's magnitude variance from to the mean ridge line determination, we have considered four clusters having low reddening and a large number of stars (NGC 0104, NGC 5024, NGC 5272, and NGC 7089). These clusters' CMDs have been randomly divided into five partial CMDs. A mean ridge line has been fitted to each partial CMD, and its MSTO's magnitude has been measured. Finally, $\sigma / \sqrt{5-1}$ for these five MSTO's measurements has been computed for each cluster, where $\sigma$ is the standard deviation of the five measurements.

Results are shown in Table 2. Column 1 shows the cluster's name, Column 2 lists $\sigma_{\mathrm{MRL}}$, and Column 3 lists $\sigma_{\text {cross-check }}=$ $\sigma / \sqrt{5-1}$ derived in this external cross-check. It can be seen that the uncertainties obtained here are equivalent to the derived $\sigma_{\mathrm{MRL}}$. This test indicates that the adopted $\sigma_{\mathrm{MRL}}$ is a good estimation.

For $\sigma_{\mathrm{MSF}}^{2}$, we adopted the uncertainty calculated by the leastsquares MS-fitting procedure. Typical values of $\sigma_{\mathrm{MSF}}$ are in the interval $\sim 0.01-0.06$ mag.

To estimate the uncertainties induced by differential reddening, binary star population, and total number of cluster stars, as well as by differences between the measured MSTO and the actual GGC's MSTO, we generated over 250 synthetic CMDs using the IAC-star (Aparicio \& Gallart 2004) synthetic CMD program. Stellar populations are calculated on a star-by-star basis. $L, T_{\text {eff }}$, and $g$ of each star are computed by direct bilogarithmic interpolation in both the metallicity and age grids of a library of stellar evolution tracks, metallicity and age following continuous distributions. All the stellar evolution phases are covered accurately, and mass loss is computed during the RGB and the asymptotic giant branch phases (see Aparicio \& Gallart 2004, for details). A set of different star formation rates, initial mass functions and metallicity laws are allowed, and binary star content can be computed. The result is a synthetic CMD with a smooth realistic stellar distribution.

Synthetic CMDs were generated with an age of $13 \mathrm{Gyr}$, [M/ $\mathrm{H}]=-1.2$, differential reddening $E(F 606 W-F 814 W)$ values between 0.0 and 0.2 , binary fractions (with $0.5<m_{2} / m_{1}<$ 1.0) between 0.0 and 0.7 , and a number of stars brighter than $M_{F 606 W}=6$ (approximately 2 mag below the MSTO) between 1000 and 50,000. The P04 stellar evolution library was used to generate the stellar libraries. For each combination of differential reddening, binary fraction and number of stars, 10 different CMDs were generated. Observational errors were simulated in all the synthetic CMDs by applying a Gaussian dispersion to the synthetic CMD, with $\sigma$ taken from the dispersion of the observed CMD as a function of the magnitude.

Using the same mean-ridge-line-fitting method used for the observational data, MSTO's magnitudes were measured for the synthetic CMD. The results are shown in Figure 4. Gray points show the MSTO measurements from the 10 different CMDs generated for each experiment, black points show the mean, and the error bars correspond to the standard deviation of the 10 measurements. Solid line represents the input MSTO magnitude, that is, the magnitude of the MSTO obtained directly from the input isochrone. It can be seen that a systematic effect on the measured MSTO's magnitudes exists. Output MSTOs (measured) are systematically $\sim 0.02$ mag fainter than input ones. This would have an effect on the measured absolute ages, but as this work is focused on relative ages, the variance of the difference between the input and output MSTO's magnitudes, $\sigma_{\mathrm{IO}}^{2}$, will be also considered.

The left panel of Figure 4 shows the $M_{F 606 W}^{\mathrm{TO}}$ measurements for the synthetic CMDs with no differential reddening, no binary stars and varying the number of stars brighter than $M_{F 606 W}=6$ from 1000 to 50,000. With the aim of determining $\sigma_{\mathrm{NUM}}^{2}$, a Gaussian has been generated for each black point, being the mean and the sigma of the Gaussian equal to the value of the corresponding point and its sigma, respectively. Finally, all Gaussians have been added together, and the variance of this sum of Gaussians has been adopted as $\sigma_{\mathrm{NUM}}^{2}$. Since most of the target clusters have more than 1000 stars brighter than $F 606 \mathrm{~W}=6$, we adopt $\sigma_{\mathrm{NUM}}=1.6 \times 10^{-2} \mathrm{mag}$ (dotted lines). Estimated this way, $\sigma_{\mathrm{NUM}}^{2}$ also includes the $\sigma_{\mathrm{IO}}^{2}$ contribution.

The central panel of Figure 4 shows the effect of differential reddening upon the estimated $M_{F 606 W}^{\mathrm{TO}}$ value. For a synthetic $\mathrm{CMD}$, the reddening has been simulated by shifting every star along the reddening vector a quantity between zero and a maximum differential reddening value, $E(F 606 W-F 814 W)_{\max }$. We generated a set of nine synthetic CMDs with 50,000 stars brighter than $F 606 W=6$ and different values of $E(F 606 W-F 814 W)_{\max }$ from 0.0 to $0.2 \mathrm{mag}$. It is worth noting that the black points' variance also includes the contribution from $\sigma_{\mathrm{IO}}^{2}$. In order to avoid duplicity, the contribution of $\sigma_{\mathrm{IO}}^{2}$ must be eliminated. As these synthetic diagrams have 50,000 stars brighter than $F 606 W=6$, their $\sigma_{\mathrm{IO}}^{2}$ is equal to the variance of the point in the left panel corresponding to a number of stars brighter than $F 606 W=6$ equal to 50,000, 


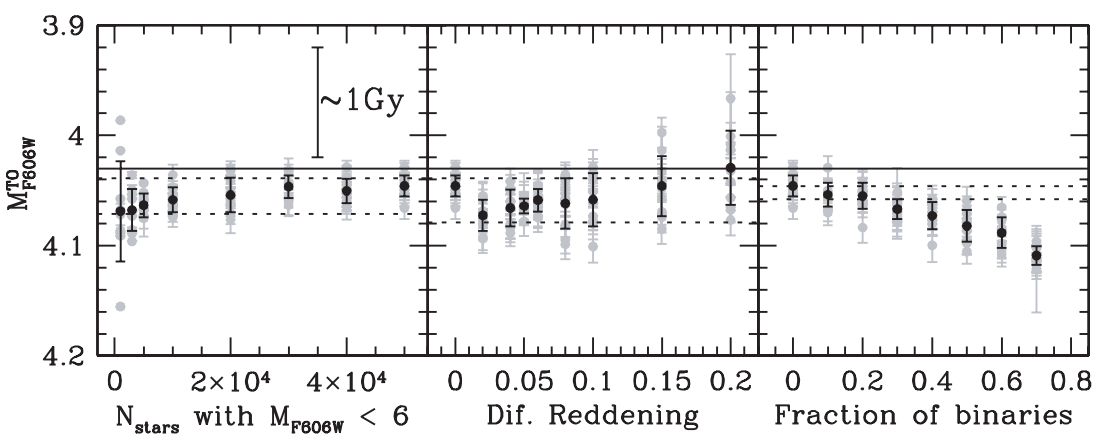

Figure 4. Right panel shows $M_{F 606 W}^{\mathrm{TO}}$ measurements over synthetic CMDs with 50,000 stars brighter than $M_{F 606 W}=6$, no differential reddening, and varying the fraction of binary stars. Grey points show the MSTO measurements for the 10 different CMDs generated in each case, and black points show the average and standard deviation of each of the 10 measurement sets. Solid line represents the MSTO's magnitude measured directly on the isochrone. The central panel shows the same as left panel, but now the fraction of binary stars is 0.0 and the maximum differential reddening is varying between 0.0 and 0.2 . The left panel shows the same, but now the fraction of binary stars is 0.0 , the differential reddening is 0.0 and the number of stars brighter then $M_{F 606 \mathrm{~W}}=6$ is varying between 1000 and 50,000 . Dashed lines represent the adopted $\pm \sigma$ in each case. See text for details.

that is $\sigma_{\mathrm{IO}}=9.7 \times 10^{-3} \mathrm{mag}$. In order to compute the effect of differential reddening upon the estimated $M_{F 606 W}^{\mathrm{TO}}$ value, $\sigma_{\mathrm{IO}}^{2}$ has been quadratically subtracted to each point's variance in the central panel of Figure 4. The resulting variance accounts for the differential reddening effect only. Then a Gaussian has been generated for each black point, being the mean and the sigma of the Gaussian equal to the value of the corresponding point and the obtained quadratically subtracted sigma, respectively. Finally, all Gaussians have been added together, and the variance of this sum of Gaussians has been adopted as $\sigma_{\mathrm{RED}}^{2}$. As we expect most of the target clusters to have differential reddening lower than $0.15 \mathrm{mag}$, we adopt $\sigma_{\mathrm{RED}}=2.0 \times 10^{-2} \mathrm{mag}$.

The right panel of Figure 4 shows the $M_{F 606 W}^{\mathrm{TO}}$ measurements of the synthetic CMDs with 50,000 stars brighter than $M_{F 606 \mathrm{~W}}=6$, no differential reddening, and varying fractions of binary stars. It can be seen that the fraction of binary stars affects $M_{F 606 W}^{\mathrm{TO}}$, making the measured MSTO fainter for clusters with a higher binary fractions. Following the same procedure as before, $\sigma_{\mathrm{IO}}^{2}$ has been quadratically subtracted to each point's variance in the right panel of Figure 4. Then a Gaussian has been generated for each black point, being the mean and the sigma of the Gaussian equal to the value of the corresponding point and the sigma obtained by subtracting $\sigma_{\mathrm{IO}}^{2}$ to the rms of the point, respectively. Finally, all Gaussians have been added together and the variance of this sum of Gaussians has been adopted as $\sigma_{\mathrm{BIN}}^{2}$. In a parallel work, we are measuring the fraction of binary stars in each cluster in our database (A. P. Milone et al. 2009, in preparation). For most clusters, it is smaller than $20 \%$. Accordingly, we consider the fraction of binary stars interval $[0.0,0.2]$ which results in $\sigma_{\mathrm{BIN}}=5.9 \times 10^{-3} \mathrm{mag}$.

To estimate the effect of cluster crowding on the measured MSTO magnitude, we used the artificial-star tests described in Anderson et al. (2008). In principle, crowding would produce some systematic shift between output and input magnitudes of artificial stars. We are interested in evaluating if these shifts significantly change as a function of crowding. Since different clusters have different crowding levels, determining the artificial star magnitude shifts in several of them will provide the information we need. We will use the NGC 0104, NGC 1851, NGC 2808, NGC 5139, NGC 5286, NGC 6171 and E3 clusters. This sample contains both one of the most and one of the least crowded clusters, namely NGC 2808 and E3, respectively.

Each cluster has associated a table containing input and output positions and magnitudes of the artificial stars. For each cluster, we selected artificial stars with input $F 814 W(I)$
Table 3

Summary of $M_{F 606 W}^{\mathrm{TO}}$ Uncertainty Contributions

\begin{tabular}{lcc}
\hline \hline Contribution & Mag. & Quadratic sum \\
\hline$\sigma_{\mathrm{MRL}}$ & $\sim 0.01-0.04$ & \\
$\sigma_{\mathrm{MSF}}$ & $\sim 0.01-0.06$ & \\
$\sigma_{\mathrm{NUM}}$ & 0.016 & \\
$\sigma_{\text {RED }}$ & 0.020 & $0.026 \mathrm{mag}$ \\
$\sigma_{\text {BIN }}$ & 0.0059 & \\
$\sigma_{\text {CROW }}$ & 0.0015 & \\
\hline
\end{tabular}

magnitudes within \pm 0.25 mag of the MSTO. For most clusters, $\sim 4000$ artificial stars fulfill this condition. For each of them, we calculated the quantity $\delta I=I_{\text {out }}-I_{\text {in }}$, where $I_{\text {out }}$ and $I_{\text {in }}$ are the recovered and input magnitudes, respectively. To have a representative estimate of $\delta I$ and also of $\sigma(\delta I)$ for each cluster, we have divided the artificial stars in 10 subsamples and calculated $\operatorname{med}(\delta I)$ for each of them. The average of medians, $\langle\operatorname{med}(\delta I)\rangle$, and the mean square root, $\sigma[\operatorname{med}(\delta I)]$, of the 10 $\operatorname{med}(\delta I)$ values have been computed for each cluster. Standard errors $\sigma_{n}=\sigma[\operatorname{med}(\delta I)] / \sqrt{(n-1)}$, with $n=10$, are obtained. These can be considered as an estimate of the internal errors of $\operatorname{med}(\delta I)$ of each cluster. Average values $\langle\operatorname{med}(\delta I)\rangle$ run from $3.0 \times 10^{-4}$ mag for E3 to $-4.5 \times 10^{-3}$ mag for NGC 2808 , while $\sigma_{n}$ is always of the order of $1.0 \times 10^{-4} \mathrm{mag}$, indicating that the $\operatorname{med}(\delta I)$ are significant. In summary, we can say that $\sim 5 \times 10^{-3}$ mag is the maximum shift introduced by crowding in our MSTO measurements. Finally, we can estimate the uncertainty produced by crowding that we were looking for, $\sigma_{\mathrm{CROW}}$, as the rms of the $\langle\operatorname{med}(\delta I)\rangle$ values of each cluster. This results in $\sigma_{\mathrm{CROW}}=1.5 \times 10^{-3} \mathrm{mag}$.

Table 3 lists a summary of the different contributions to $M_{F 606 W}^{\mathrm{TO}}$ uncertainties. The MSTO's magnitude variances produced by the cluster number of stars, differential reddening, binary stars content and crowding, evaluated in this section using simulations, have been quadratically added and the resulting sigma (0.026 mag) is also shown in Table 3. Finally, $M_{F 606 \mathrm{~W}}^{\mathrm{TO}}$ uncertainty has been evaluated for each cluster adding quadratically 0.026 mag to the obtained $\sigma_{\mathrm{MRL}}^{2}$ and $\sigma_{\mathrm{MSF}}^{2}$ (Equation (2)), and the results are listed in Table 4 (Column 4). It is worth mentioning that the total uncertainties tend to increase both at low and high metallicities. This is due to the error propagation in the $\sigma_{\mathrm{MSF}}^{2}$ contribution, as we are using the intermediate metallicity cluster NGC 6752 for the MSTO absolute magnitude zero point. 
Table 4

Globular Cluster Relative Ages

\begin{tabular}{|c|c|c|c|c|c|c|c|c|c|}
\hline Name & {$[\mathrm{M} / \mathrm{H}]_{\mathrm{ZW}}$} & {$[\mathrm{M} / \mathrm{H}]_{\mathrm{CG}}$} & $M_{F 606 W}^{\mathrm{TO}}$ & $\mathrm{D} 07_{\mathrm{ZW}}$ & $\mathrm{D} 07_{\mathrm{CG}}$ & $\mathrm{P} 04_{\mathrm{CG}}$ & B94 $4_{\mathrm{CG}}$ & $\mathrm{G} 00_{\mathrm{CG}}$ & Group \\
\hline NGC 0104 & -0.57 & -0.64 & $4.13 \pm 0.07$ & $0.96 \pm 0.07$ & $1.02 \pm 0.07$ & $1.07 \pm 0.08$ & $1.02 \pm 0.07$ & $1.05 \pm 0.09$ & Old \\
\hline NGC 0288 & -1.18 & -0.92 & $3.82 \pm 0.04$ & $0.88 \pm 0.04$ & $0.83 \pm 0.03$ & $0.83 \pm 0.03$ & $0.82 \pm 0.03$ & $0.83 \pm 0.03$ & Young \\
\hline NGC 0362 & -1.11 & -0.87 & $3.81 \pm 0.05$ & $0.84 \pm 0.04$ & $0.81 \pm 0.04$ & $0.80 \pm 0.04$ & $0.81 \pm 0.04$ & $0.81 \pm 0.05$ & Young \\
\hline NGC 1261 & -1.10 & -0.86 & $3.80 \pm 0.05$ & $0.83 \pm 0.04$ & $0.80 \pm 0.04$ & $0.79 \pm 0.04$ & $0.80 \pm 0.04$ & $0.79 \pm 0.05$ & Young \\
\hline NGC 1851 & -1.01 & -0.81 & $3.79 \pm 0.05$ & $0.80 \pm 0.04$ & $0.78 \pm 0.04$ & $0.77 \pm 0.04$ & $0.78 \pm 0.04$ & $0.75 \pm 0.04$ & Young \\
\hline NGC 2298 & -1.69 & -1.49 & $3.80 \pm 0.05$ & $1.01 \pm 0.05$ & $0.99 \pm 0.05$ & $0.99 \pm 0.05$ & $0.99 \pm 0.05$ & $0.99 \pm 0.05$ & Old \\
\hline NGC 2808 & -1.14 & -0.89 & $3.86 \pm 0.03$ & $0.91 \pm 0.03$ & $0.85 \pm 0.03$ & $0.85 \pm 0.03$ & $0.85 \pm 0.03$ & $0.85 \pm 0.02$ & Young \\
\hline NGC 3201 & -1.31 & -1.02 & $3.75 \pm 0.04$ & $0.86 \pm 0.03$ & $0.80 \pm 0.03$ & $0.79 \pm 0.03$ & $0.80 \pm 0.02$ & $0.81 \pm 0.03$ & Young \\
\hline NGC 4147 & -1.55 & -1.28 & $3.74 \pm 0.04$ & $0.93 \pm 0.04$ & $0.89 \pm 0.04$ & $0.88 \pm 0.04$ & $0.89 \pm 0.04$ & $0.89 \pm 0.03$ & Young \\
\hline NGC 4590 & -1.89 & -1.78 & $3.61 \pm 0.05$ & $0.89 \pm 0.03$ & $0.90 \pm 0.04$ & $0.89 \pm 0.04$ & $0.90 \pm 0.04$ & $0.91 \pm 0.04$ & Old \\
\hline NGC 4833 & -1.70 & -1.49 & $3.80 \pm 0.05$ & $1.01 \pm 0.05$ & $0.98 \pm 0.05$ & $0.98 \pm 0.04$ & $0.98 \pm 0.05$ & $0.98 \pm 0.05$ & Old \\
\hline NGC 5024 & -1.82 & -1.64 & $3.76 \pm 0.05$ & $1.01 \pm 0.05$ & $0.99 \pm 0.05$ & $0.99 \pm 0.04$ & $1.00 \pm 0.05$ & $0.99 \pm 0.05$ & Old \\
\hline NGC 5053 & -1.88 & -1.76 & $3.69 \pm 0.05$ & $0.95 \pm 0.04$ & $0.96 \pm 0.04$ & $0.97 \pm 0.04$ & $0.96 \pm 0.05$ & $0.96 \pm 0.04$ & Old \\
\hline NGC 5139 & -1.37 & -1.13 & $3.82 \pm 0.05$ & $0.94 \pm 0.05$ & $0.90 \pm 0.05$ & $0.89 \pm 0.05$ & $0.90 \pm 0.05$ & $0.89 \pm 0.05$ & Young \\
\hline NGC 5272 & -1.44 & -1.12 & $3.83 \pm 0.04$ & $0.96 \pm 0.04$ & $0.89 \pm 0.04$ & $0.88 \pm 0.04$ & $0.90 \pm 0.03$ & $0.89 \pm 0.04$ & Young \\
\hline NGC 5286 & -1.48 & -1.19 & $3.89 \pm 0.05$ & $1.04 \pm 0.04$ & $0.98 \pm 0.04$ & $0.98 \pm 0.05$ & $0.97 \pm 0.05$ & $0.98 \pm 0.05$ & Old \\
\hline NGC 5466 & -2.00 & -1.98 & $3.74 \pm 0.05$ & $1.03 \pm 0.05$ & $1.06 \pm 0.05$ & $1.06 \pm 0.06$ & $1.08 \pm 0.03$ & $1.07 \pm 0.05$ & Old \\
\hline NGC 5904 & -1.16 & -0.90 & $3.83 \pm 0.03$ & $0.88 \pm 0.03$ & $0.83 \pm 0.03$ & $0.83 \pm 0.03$ & $0.83 \pm 0.03$ & $0.83 \pm 0.02$ & Young \\
\hline NGC 5927 & -0.18 & -0.50 & $4.15 \pm 0.07$ & $0.88 \pm 0.06$ & $0.99 \pm 0.07$ & $1.03 \pm 0.08$ & $0.98 \pm 0.07$ & $1.01 \pm 0.11$ & Old \\
\hline NGC 5986 & -1.43 & -1.13 & $3.88 \pm 0.05$ & $1.01 \pm 0.04$ & $0.95 \pm 0.04$ & $0.94 \pm 0.05$ & $0.93 \pm 0.04$ & $0.95 \pm 0.05$ & Old \\
\hline NGC 6093 & -1.53 & -1.25 & $3.87 \pm 0.05$ & $1.03 \pm 0.04$ & $0.98 \pm 0.04$ & $0.98 \pm 0.05$ & $0.97 \pm 0.05$ & $0.98 \pm 0.05$ & Old \\
\hline NGC 6101 & -1.73 & -1.54 & $3.78 \pm 0.04$ & $1.00 \pm 0.04$ & $0.98 \pm 0.04$ & $0.98 \pm 0.04$ & $0.98 \pm 0.04$ & $0.98 \pm 0.04$ & Old \\
\hline NGC 6121 & -1.05 & -0.83 & $4.01 \pm 0.05$ & $1.02 \pm 0.05$ & $0.98 \pm 0.05$ & $0.99 \pm 0.07$ & $0.97 \pm 0.05$ & $0.98 \pm 0.05$ & Old \\
\hline NGC 6144 & -1.71 & -1.52 & $3.88 \pm 0.05$ & $1.10 \pm 0.05$ & $1.08 \pm 0.05$ & $1.07 \pm 0.06$ & $1.05 \pm 0.05$ & $1.07 \pm 0.05$ & Old \\
\hline NGC 6171 & -0.87 & -0.81 & $4.14 \pm 0.05$ & $1.08 \pm 0.05$ & $1.09 \pm 0.06$ & $1.11 \pm 0.06$ & $1.07 \pm 0.06$ & $1.13 \pm 0.08$ & Old \\
\hline NGC 6205 & -1.41 & -1.11 & $3.84 \pm 0.04$ & $0.97 \pm 0.04$ & $0.91 \pm 0.04$ & $0.90 \pm 0.04$ & $0.90 \pm 0.02$ & $0.90 \pm 0.04$ & Young \\
\hline NGC 6218 & -1.18 & -0.92 & $3.99 \pm 0.04$ & $1.05 \pm 0.04$ & $0.99 \pm 0.03$ & $1.00 \pm 0.05$ & $0.99 \pm 0.04$ & $0.99 \pm 0.03$ & Old \\
\hline NGC 6254 & -1.33 & -1.03 & $3.86 \pm 0.04$ & $0.96 \pm 0.04$ & $0.89 \pm 0.04$ & $0.88 \pm 0.04$ & $0.90 \pm 0.03$ & $0.88 \pm 0.04$ & Young \\
\hline NGC 6304 & -0.24 & -0.52 & $4.19 \pm 0.07$ & $0.93 \pm 0.06$ & $1.06 \pm 0.08$ & $1.09 \pm 0.09$ & $1.05 \pm 0.07$ & $1.09 \pm 0.10$ & Old \\
\hline NGC 6341 & -2.02 & -1.94 & $3.72 \pm 0.04$ & $1.02 \pm 0.04$ & $1.03 \pm 0.04$ & $1.03 \pm 0.05$ & $1.05 \pm 0.04$ & $1.03 \pm 0.05$ & Old \\
\hline NGC 6352 & -0.36 & -0.56 & $4.13 \pm 0.07$ & $0.90 \pm 0.06$ & $0.99 \pm 0.07$ & $1.03 \pm 0.08$ & $0.99 \pm 0.07$ & $1.02 \pm 0.10$ & Old \\
\hline NGC 6362 & -0.96 & -0.85 & $4.09 \pm 0.05$ & $1.06 \pm 0.05$ & $1.06 \pm 0.05$ & $1.08 \pm 0.05$ & $1.04 \pm 0.03$ & $1.07 \pm 0.06$ & Old \\
\hline NGC 6366 & -0.44 & -0.59 & $4.16 \pm 0.12$ & $0.95 \pm 0.07$ & $1.04 \pm 0.13$ & $1.08 \pm 0.16$ & $1.04 \pm 0.13$ & $1.07 \pm 0.18$ & Old \\
\hline NGC 6388 & -0.60 & -0.63 & $4.05 \pm 0.08$ & $0.90 \pm 0.07$ & $0.94 \pm 0.08$ & $0.95 \pm 0.10$ & $0.92 \pm 0.07$ & $0.94 \pm 0.10$ & Old \\
\hline NGC 6397 & -1.72 & -1.54 & $3.79 \pm 0.04$ & $1.01 \pm 0.04$ & $0.99 \pm 0.04$ & $0.99 \pm 0.04$ & $0.99 \pm 0.04$ & $0.99 \pm 0.04$ & Old \\
\hline NGC 6441 & -0.45 & -0.46 & $4.04 \pm 0.08$ & $0.85 \pm 0.06$ & $0.88 \pm 0.07$ & $0.90 \pm 0.07$ & $0.88 \pm 0.05$ & $0.85 \pm 0.09$ & Old \\
\hline NGC 6496 & -0.36 & -0.56 & $4.11 \pm 0.07$ & $0.88 \pm 0.06$ & $0.97 \pm 0.07$ & $1.00 \pm 0.09$ & $0.95 \pm 0.07$ & $0.98 \pm 0.10$ & Old \\
\hline NGC 6535 & -1.56 & -1.29 & $3.68 \pm 0.11$ & $0.87 \pm 0.04$ & $0.82 \pm 0.09$ & $0.82 \pm 0.09$ & $0.81 \pm 0.09$ & $0.83 \pm 0.08$ & Young \\
\hline NGC 6541 & -1.57 & -1.31 & $3.88 \pm 0.04$ & $1.06 \pm 0.04$ & $1.01 \pm 0.04$ & $1.02 \pm 0.04$ & $1.00 \pm 0.03$ & $1.01 \pm 0.04$ & Old \\
\hline NGC 6584 & -1.32 & -1.10 & $3.82 \pm 0.03$ & $0.92 \pm 0.03$ & $0.88 \pm 0.03$ & $0.87 \pm 0.03$ & $0.89 \pm 0.03$ & $0.87 \pm 0.03$ & Young \\
\hline NGC 6624 & -0.36 & -0.56 & $4.12 \pm 0.07$ & $0.89 \pm 0.06$ & $0.98 \pm 0.07$ & $1.01 \pm 0.08$ & $0.98 \pm 0.07$ & $1.00 \pm 0.10$ & Old \\
\hline NGC 6637 & -0.58 & -0.64 & $4.13 \pm 0.07$ & $0.96 \pm 0.07$ & $1.02 \pm 0.07$ & $1.07 \pm 0.08$ & $1.02 \pm 0.07$ & $1.05 \pm 0.09$ & Old \\
\hline NGC 6652 & -0.75 & -0.76 & $4.07 \pm 0.06$ & $0.97 \pm 0.06$ & $1.01 \pm 0.06$ & $1.03 \pm 0.08$ & $1.01 \pm 0.06$ & $1.03 \pm 0.08$ & Old \\
\hline NGC 6656 & -1.53 & -1.27 & $3.87 \pm 0.05$ & $1.03 \pm 0.05$ & $0.99 \pm 0.05$ & $0.99 \pm 0.05$ & $0.98 \pm 0.05$ & $0.98 \pm 0.05$ & Old \\
\hline NGC 6681 & -1.42 & -1.13 & $3.93 \pm 0.04$ & $1.07 \pm 0.04$ & $1.00 \pm 0.04$ & $1.01 \pm 0.04$ & $1.00 \pm 0.03$ & $1.01 \pm 0.04$ & Old \\
\hline NGC 6715 & -1.32 & -1.03 & $3.79 \pm 0.03$ & $0.90 \pm 0.03$ & $0.84 \pm 0.03$ & $0.83 \pm 0.03$ & $0.83 \pm 0.04$ & $0.84 \pm 0.02$ & Young \\
\hline NGC 6717 & -1.11 & -0.87 & $4.06 \pm 0.05$ & $1.09 \pm 0.05$ & $1.03 \pm 0.05$ & $1.07 \pm 0.05$ & $1.03 \pm 0.04$ & $1.04 \pm 0.06$ & Old \\
\hline NGC 6723 & -0.90 & -0.82 & $4.07 \pm 0.05$ & $1.02 \pm 0.05$ & $1.02 \pm 0.05$ & $1.06 \pm 0.05$ & $1.02 \pm 0.04$ & $1.04 \pm 0.06$ & Old \\
\hline NGC 6752 & -1.32 & -1.02 & $3.88 \pm 0.03$ & $0.98 \pm 0.03$ & $0.92 \pm 0.04$ & $0.91 \pm 0.03$ & $0.91 \pm 0.02$ & $0.92 \pm 0.04$ & Young \\
\hline NGC 6779 & -1.72 & -1.50 & $3.89 \pm 0.05$ & $1.10 \pm 0.05$ & $1.07 \pm 0.05$ & $1.06 \pm 0.06$ & $1.05 \pm 0.04$ & $1.06 \pm 0.04$ & Old \\
\hline NGC 6809 & -1.58 & -1.32 & $3.83 \pm 0.04$ & $1.01 \pm 0.04$ & $0.96 \pm 0.04$ & $0.96 \pm 0.05$ & $0.95 \pm 0.04$ & $0.96 \pm 0.05$ & Old \\
\hline NGC 6838 & -0.44 & -0.59 & $4.19 \pm 0.07$ & $0.98 \pm 0.08$ & $1.07 \pm 0.08$ & $1.10 \pm 0.10$ & $1.06 \pm 0.08$ & $1.11 \pm 0.10$ & Old \\
\hline NGC 6934 & -1.32 & -1.10 & $3.81 \pm 0.04$ & $0.91 \pm 0.04$ & $0.87 \pm 0.04$ & $0.86 \pm 0.04$ & $0.88 \pm 0.04$ & $0.86 \pm 0.03$ & Young \\
\hline NGC 6981 & -1.28 & -0.99 & $3.82 \pm 0.03$ & $0.91 \pm 0.02$ & $0.85 \pm 0.02$ & $0.84 \pm 0.02$ & $0.85 \pm 0.03$ & $0.85 \pm 0.02$ & Young \\
\hline NGC 7078 & -1.91 & -1.80 & $3.74 \pm 0.05$ & $1.00 \pm 0.04$ & $1.01 \pm 0.04$ & $1.01 \pm 0.04$ & $1.02 \pm 0.04$ & $1.01 \pm 0.04$ & Old \\
\hline NGC 7089 & -1.39 & -1.09 & $3.86 \pm 0.04$ & $0.98 \pm 0.04$ & $0.92 \pm 0.04$ & $0.91 \pm 0.04$ & $0.91 \pm 0.03$ & $0.92 \pm 0.05$ & Young \\
\hline NGC 7099 & -1.83 & -1.70 & $3.76 \pm 0.05$ & $1.01 \pm 0.04$ & $1.01 \pm 0.05$ & $1.01 \pm 0.04$ & $1.01 \pm 0.04$ & $1.01 \pm 0.04$ & Old \\
\hline ARP 2 & -1.52 & -1.23 & $3.74 \pm 0.07$ & $0.91 \pm 0.06$ & $0.85 \pm 0.06$ & $0.84 \pm 0.06$ & $0.84 \pm 0.06$ & $0.85 \pm 0.05$ & Young \\
\hline E3 & -0.66 & -0.69 & $4.09 \pm 0.11$ & $0.95 \pm 0.05$ & $1.00 \pm 0.11$ & $1.02 \pm 0.13$ & $1.00 \pm 0.10$ & $1.02 \pm 0.15$ & Old \\
\hline LYNGÅ7 & -0.48 & -0.50 & $4.27 \pm 0.12$ & $1.09 \pm 0.08$ & $1.13 \pm 0.14$ & $1.18 \pm 0.14$ & $1.10 \pm 0.15$ & $1.19 \pm 0.18$ & Old \\
\hline PAL 1 & -0.46 & -0.56 & $3.59 \pm 0.14$ & $0.54 \pm 0.06$ & $0.57 \pm 0.09$ & $0.59 \pm 0.05$ & $0.63 \pm 0.07$ & $0.55 \pm 0.10$ & Young \\
\hline PAL 12 & -0.68 & -0.69 & $3.73 \pm 0.11$ & $0.67 \pm 0.04$ & $0.69 \pm 0.09$ & $0.69 \pm 0.08$ & $0.72 \pm 0.07$ & $0.68 \pm 0.07$ & Young \\
\hline TERZAN7 & -0.44 & -0.42 & $3.63 \pm 0.07$ & $0.55 \pm 0.04$ & $0.57 \pm 0.04$ & $0.57 \pm 0.05$ & $0.63 \pm 0.04$ & $0.51 \pm 0.05$ & Young \\
\hline TERZAN8 & -1.75 & -1.58 & $3.73 \pm 0.05$ & $0.96 \pm 0.04$ & $0.95 \pm 0.04$ & $0.95 \pm 0.04$ & $0.94 \pm 0.05$ & $0.95 \pm 0.04$ & Old \\
\hline
\end{tabular}



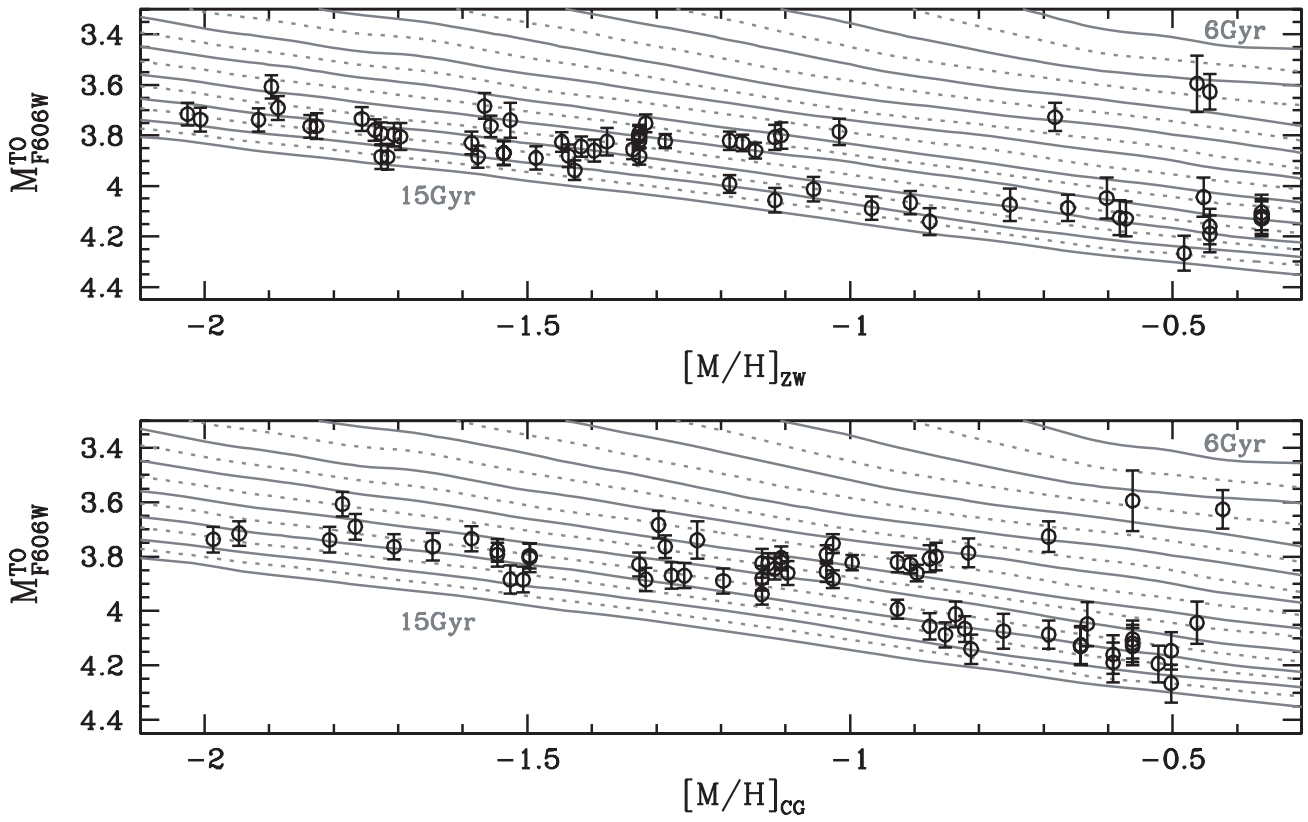

Figure 5. Theoretical TO absolute magnitudes from the D07 stellar evolution library. Lines represent $M_{F 606 W}^{\mathrm{TO}}$ for different ages as a function of global metallicity, in $1 \mathrm{Gyr}$ (solid lines) and $0.5 \mathrm{Gyr}$ (dashed lines) age steps. The lower isochrone corresponds to an age of 15 Gyr. Points represent the measured MSTO magnitudes. The upper panel shows the $M_{F 606 W}^{\mathrm{TO}}$ magnitude as a function of the metallicity in the $\mathrm{ZW}$ scale; the lower panel gives $M_{F 606 W}^{\mathrm{TO}}$ as a function of the metallicity in the CG scale.

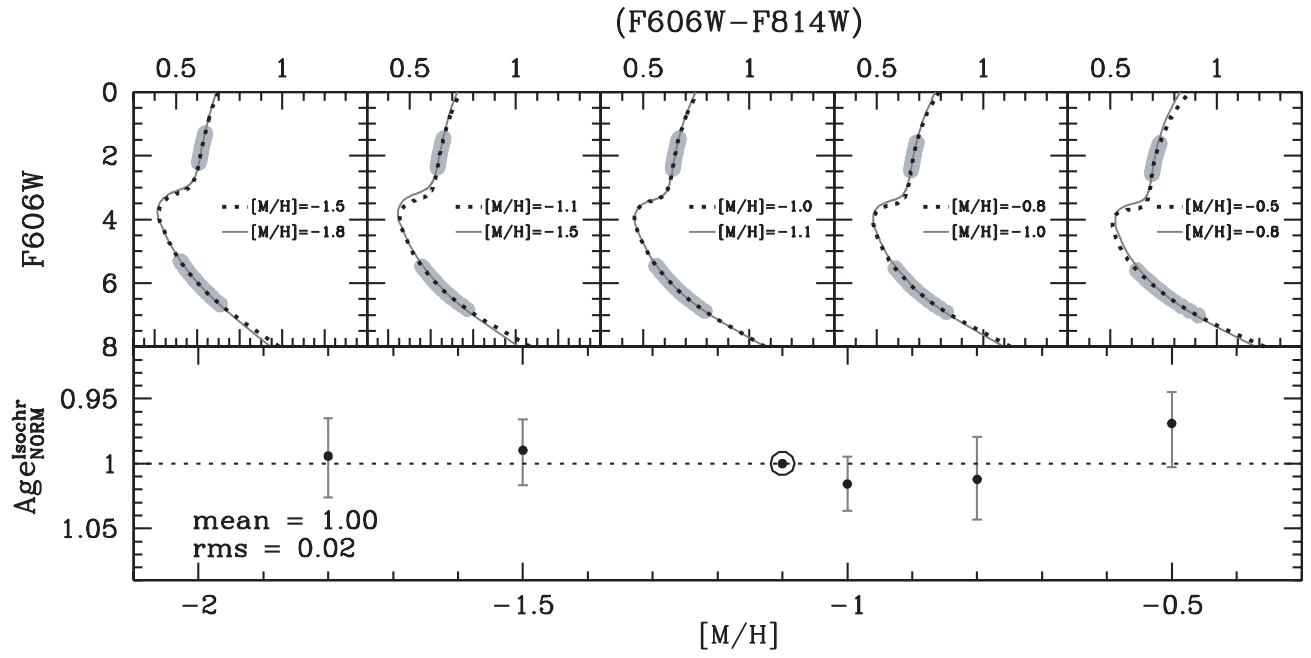

Figure 6. Testing the used MS-fitting procedure and relative ages determination using 13 Gyr synthetic isochrones. Upper panel shows the MS fitting for 13 Gyr synthetic isochrones with the same metallicities as the adopted reference clusters $([\mathrm{M} / \mathrm{H}]=-0.5,-0.8,-1.0,-1.1,-1.5$, and -1.8$)$. Lower panel shows the obtained relative ages. The MSTO magnitude of the $[\mathrm{M} / \mathrm{H}]=-1.1$ isochrone (encircled point) has been considered as MSTO zero point.

\section{RESULTS}

In this section, we present the relative ages derived for the sample of 64 GGCs. We compare the derived ages to the theoretical isochrones of D07 using both the ZW and CG abundance scales. In the second part of this section, we test the MS-fitting procedure and check the self-consistency of the relative age determination. The relative age results are then compared with those derived using the stellar evolution libraries of P04, B94, and G00. Finally, we present a comparison of our results with previous work.

\subsection{Relative Ages}

Relative ages were calculated using a stellar evolution library (D07) to calculate the theoretical $M_{F 606 W}^{\mathrm{TO}}$ values for different ages and $[\mathrm{M} / \mathrm{H}]$. Figure 5 shows the $M_{F 606 W}^{\mathrm{TO}}$ resulting from the D07 models as a function of $[\mathrm{M} / \mathrm{H}]$, using both $\mathrm{ZW}$ and CG metallicity scales. Lines represent the D07 model MSTO magnitudes in steps of $1 \mathrm{Gyr}$ (solid lines) and $0.5 \mathrm{Gyr}$ (dotted lines). The curves are interpolated using a spline curve so that we can easily estimate $M_{F 606 \mathrm{~W}}^{\mathrm{TO}}=f([\mathrm{M} / \mathrm{H}]$, age $)$. We overplot the turnoff magnitudes calculated in Section 4.2 (open circles). The GGC metallicities listed in Table 1 have been transformed into global metallicities, $[\mathrm{M} / \mathrm{H}]$, using the prescription of Salaris et al. (1993):

$$
[\mathrm{M} / \mathrm{H}]=[\mathrm{Fe} / \mathrm{H}]+\log (0.638 f+0.362)
$$

where $\log f=[\alpha / \mathrm{Fe}]$. For the $\alpha$-element enhancement, based on previous literature estimates (Carney 1996; Salaris \& Cassisi 1996; Venn et al. 2004; Kirby et al. 2008), we assumed: 

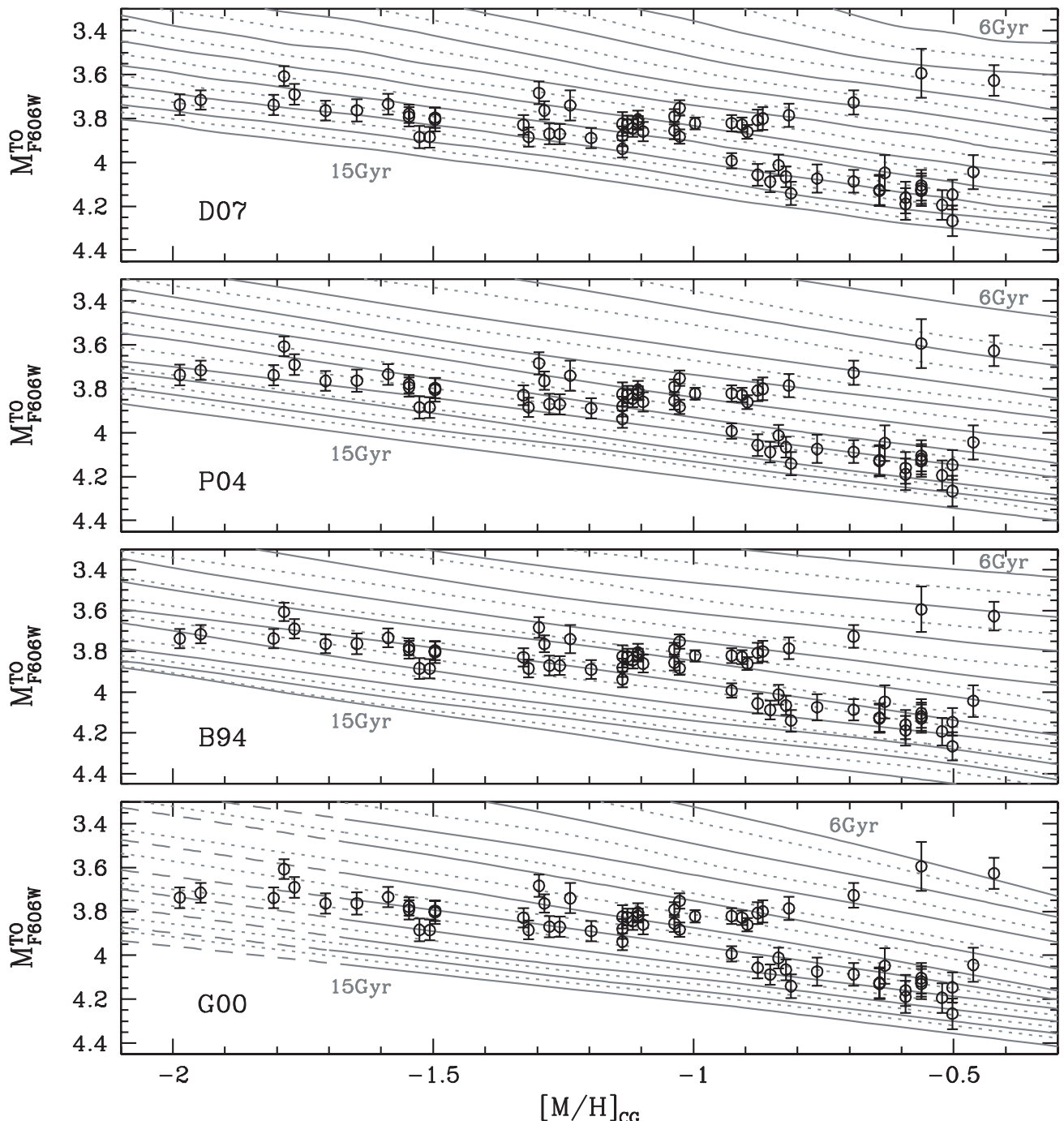

Figure 7. MSTO magnitudes predicted by the D07, P04, B94, and G00 stellar evolution libraries. Lines represent $M_{F 606 W}^{\mathrm{TO}}$ for different ages as a function of global metallicity, in $1 \mathrm{Gyr}$ (solid lines) and $0.5 \mathrm{Gyr}$ (dashed lines) age steps. The lower isochrone corresponds to an age of $15 \mathrm{Gyr}$ and the upper one to $6 \mathrm{Gyr}$. In the case of G00 models, metallicities lower than $Z=0.0004$ have been extrapolated. Points represent observational data.

$[\alpha / \mathrm{Fe}]=+0.3 \pm 0.05$ for clusters with $[\mathrm{Fe} / \mathrm{H}]<-1.0$, and $[\alpha / \mathrm{Fe}]=+0.2 \pm 0.05$ for clusters with $[\mathrm{Fe} / \mathrm{H}] \geqslant-1.0$. The effect that this \pm 0.05 dex uncertainty has on the final relative ages will be discussed at the end of this section. The values of $[\mathrm{M} / \mathrm{H}]$ in both $\mathrm{ZW}$ and $\mathrm{CG}$ scales are listed in Columns 2 and 3 in Table 4, respectively. We finally estimate the age of each cluster based on the interpolated curves.

While this procedure provides absolute ages for all of our clusters, it must be noted that absolute ages depend on the theoretical model as well as the adopted MSTO magnitude zero point. A much more detailed analysis is required to derive reliable absolute ages, and this will be done in a forthcoming paper. This paper concentrates only on relative ages, which are much less dependent on the MSTO zero point.

In order to derive relative ages, we divided the target clusters into three groups: low-metallicity $([\mathrm{M} / \mathrm{H}]<-1.4)$, intermediate-metallicity $(-1.4<[\mathrm{M} / \mathrm{H}]<-0.8)$, and highmetallicity $([\mathrm{M} / \mathrm{H}]>-0.8)$. Using the D07 models, we compute the mean age of the low-metallicity group as $12.80 \pm 0.17$ Gyr. The rms scatter of the 13 low-metallicity GGCs is equal to $0.6 \mathrm{Gyr}$. The absolute age of each cluster was then divided by this mean age, obtaining what we will call normalized age from here on. Columns 5 and 6 of Table 4 list the normalized ages from the D07 models using the ZW- and CG-metallicity scales, respectively.

The adopted \pm 0.05 dex uncertainty for the $\alpha$-element enhancement translates into an uncertainty of $\sim 0.012$ on the final relative age. With the aim of taking into consideration this effect on the relative ages, this quantity has been added quadratically to obtain the final relative age uncertainties listed in Table 4.

\subsection{Testing Self-Consistency}

In this section, the self-consistency of the MS-fitting procedure and relative age determination is tested. In particular, we are interested in quantifying the validity of matching up clusters with different metallicities on the grounds of theoretical stellar evolution models.

We created a set of $13 \mathrm{Gyr}$ synthetic isochrones using the D07 models with metallicities similar to those of the selected reference clusters NGC 6304, NGC 6723, NGC 6981, NGC 6681, NGC 6101 and NGC 4590. That is, isochrones with $[\mathrm{M} / \mathrm{H}]=-0.5,-0.8,-1.0,-1.1,-1.5$, and -1.8 have been considered. We applied the same MS-fitting procedure to the 

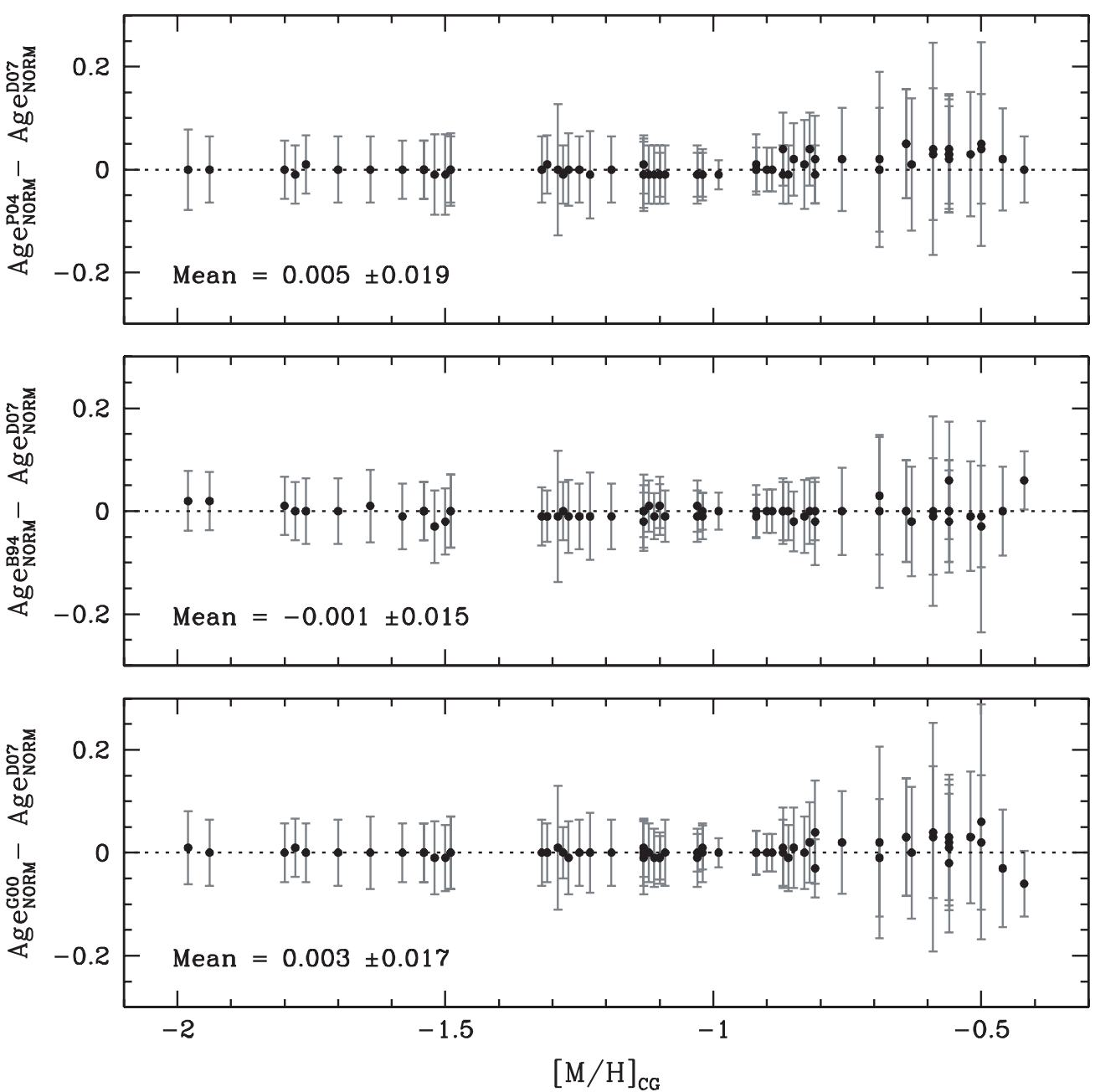

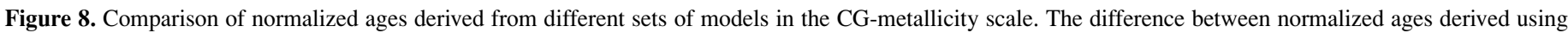

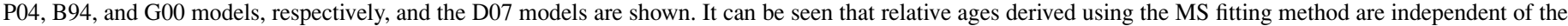
adopted model.

isochrones as for the observational data (Section 4.2). The [M/ $\mathrm{H}]=-1.1$ isochrone, located in the center of the metallicity interval, was chosen for the MSTO zero-point determination.

Figure 6 summarizes the test results. The upper panel shows the MS fitting for the $13 \mathrm{Gyr}$ synthetic isochrones with the same metallicities as the adopted reference clusters. Lower panel shows the obtained relative ages results. The MSTO of the [M/ $\mathrm{H}]=-1.1$ isochrone, that has been adopted as MSTO zero point, has been encircled. Error bars represent the uncertainties derived from the MS fitting, $\sigma_{\mathrm{MSF}}$. It can be seen that the MSfitting procedure is self-consistent, and that the derived ages are similar to the input ages with a typical uncertainty of less than $2 \%$ over the entire metallicity range. We thus conclude that our metallicity grouping should not induce a significant bias in the relative age estimation.

\subsection{Comparison to Other Stellar Evolution Libraries}

Figure 7 shows the theoretical MSTO magnitudes derived using P04, B94, and G00 stellar evolution libraries. D07 model predictions have also been plotted for comparison. Lines represent $M_{F 606 W}^{\mathrm{TO}}$ for different ages as a function of global metallicity, in 1 Gyr (solid lines) and 0.5 Gyr (dashed lines) age steps. Points represent our measured MSTO magnitudes as a function of metallicity in the CG scale. In the case of
G00 models, metallicities lower than $Z=0.0004$ have been extrapolated.

Using these theoretical grids, normalized ages have been derived following the procedure described in Section 6.1. The corresponding average age of the low metallicity group, as derived from each isochrone set, has been used for normalization.

Columns 7-9 of Table 4 list the normalized ages derived from the P04, B94, and G00 stellar evolution libraries, respectively, using the CG-metallicity scale. Figure 8 shows the difference between the normalized ages from the D07 models, and those from the P04, B94, and G00 models, adopting the CGmetallicity scale. The error bars are the quadrature of the relative age's uncertainties form the two corresponding models. Interestingly, the relatives ages are the same, within the uncertainties, independent of the adopted theoretical library.

\subsection{Comparison to Previous Studies}

Figure 9 shows a comparison of our results to previous work. The upper and middle panels show the difference between our results and the normalized ages published by De Angeli et al. (2005) using HST snapshot-deA05S-and ground-baseddeA05G-data. The lower panel shows the difference between our results (MF08) and Rosenberg et al. (1999)'s normalized 

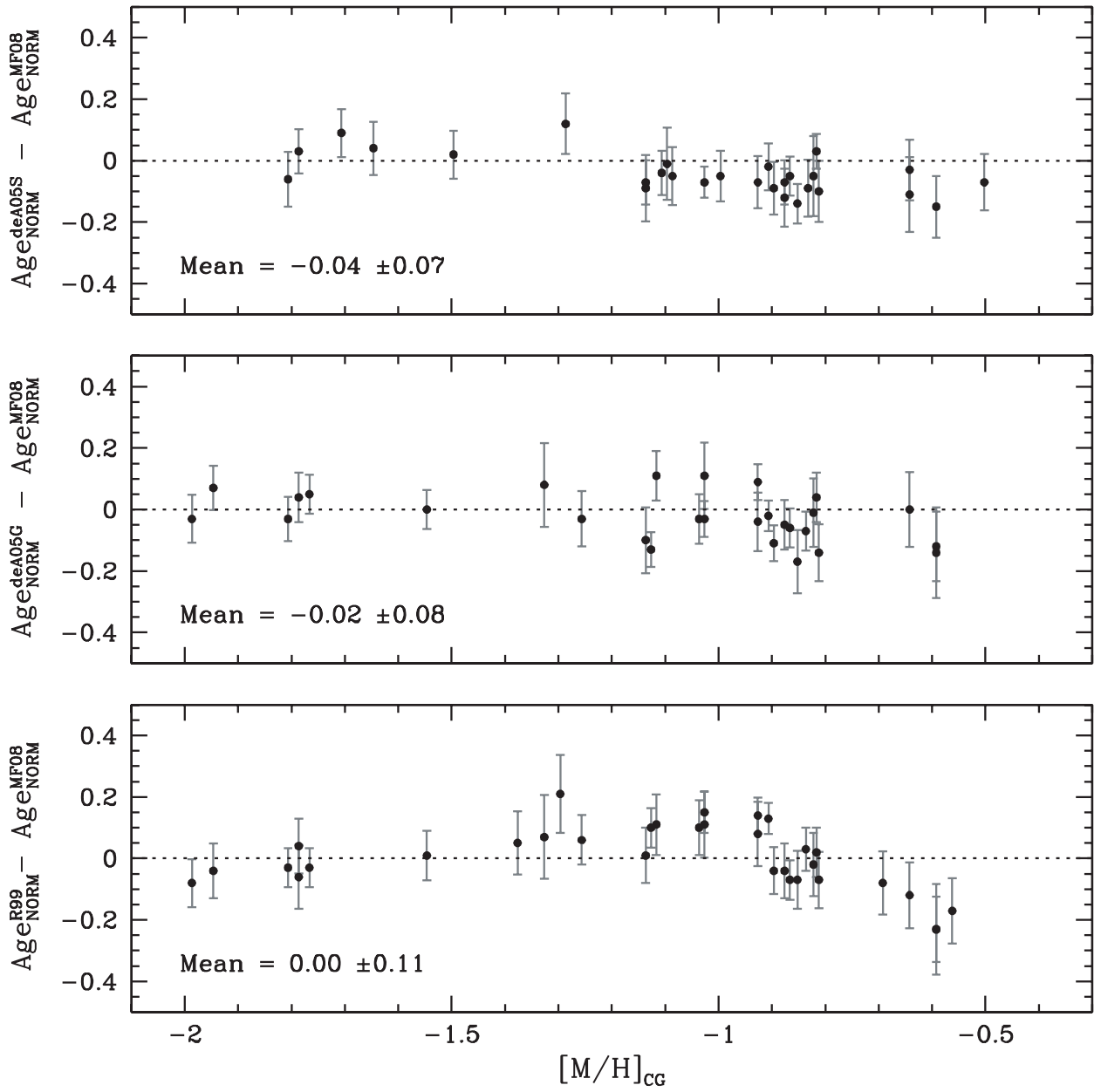

Figure 9. Comparison with previous works using the CG-metallicity scale. The differences between the normalized ages of De Angeli et al. (2005) (using HST snapshot—deA05S—and ground-based—deA05G—data) and Rosenberg et al. (1999) (R99) and the results derived in this paper by using D07 models (MF08) are indicated.
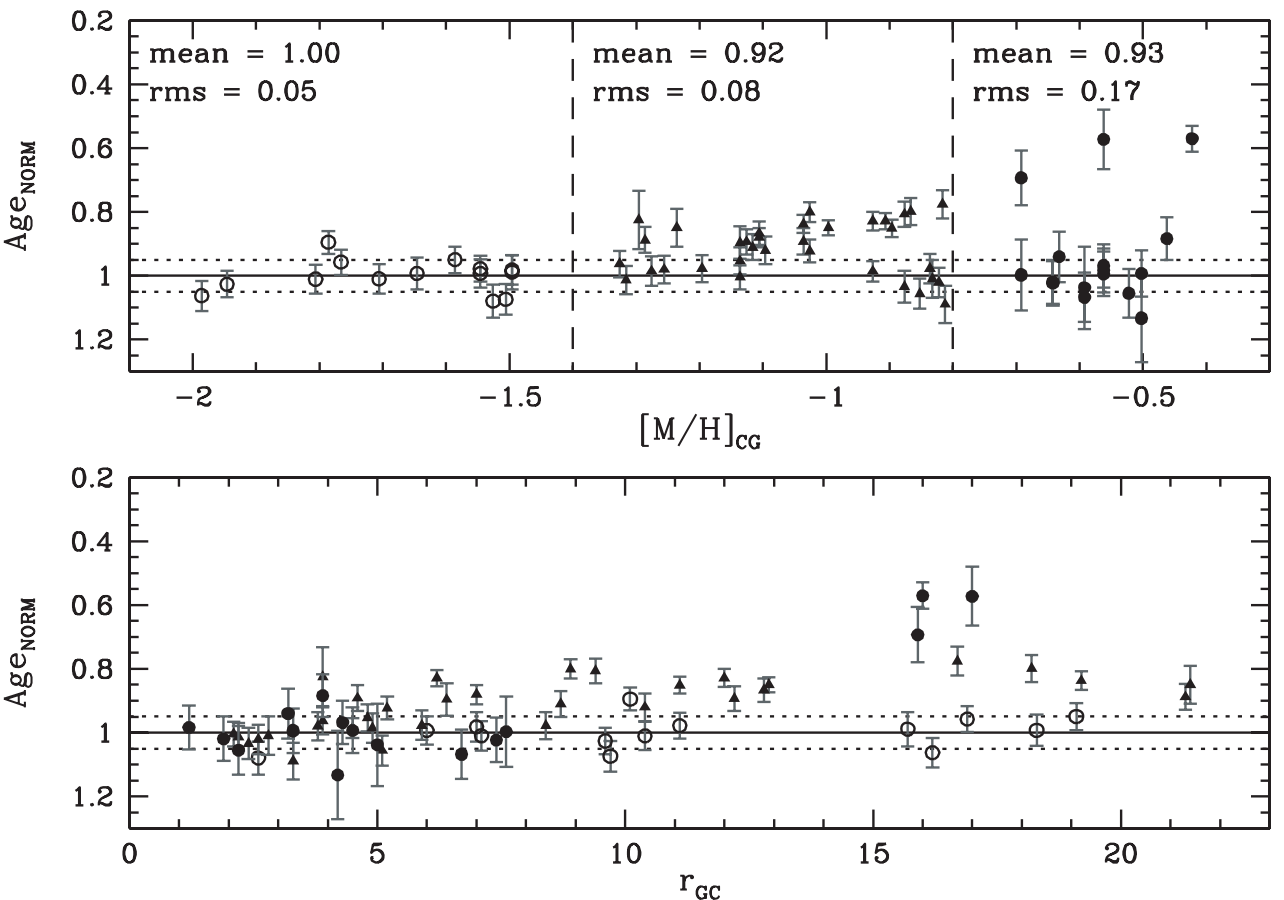

Figure 10. Globular cluster normalized ages as a function of $[\mathrm{M} / \mathrm{H}]$ in the CG-metallicity scale (upper panel), and as a function of galactocentric distance ( $r_{\mathrm{GC}}$ kpc, lower panel). These results have been derived using the D07 stellar evolution library. Open circles, filled triangles, and filled circles represent GCs within the low-, intermediate-, and high-metallicity groups, respectively. For each of the three metallicity groups, mean age and rms are indicated. See text for details. 

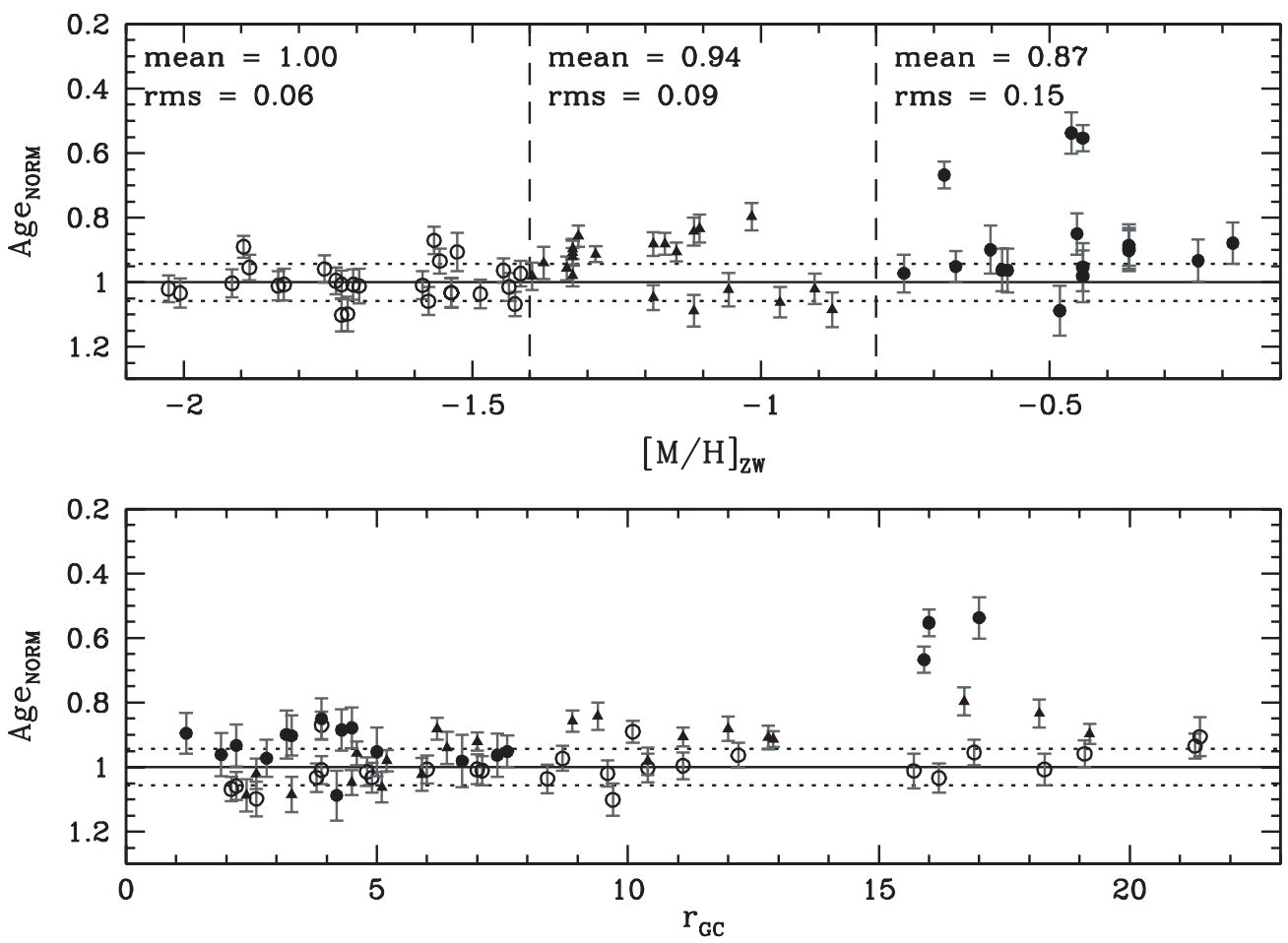

Figure 11. Same as Figure 10, but for the ZW-metallicity scale.

ages. The uncertainties are the quadrature of our listed uncertainties and those published in the De Angeli et al. (2005) and Rosenberg et al. (1999) studies. The De Angeli et al. (2005) ages are consistent with ours within the error bars and no appreciable age trend is observed. On the other hand, some trend is seen when comparing our results to those by Rosenberg et al. (1999),who find marginally older ages for cluster with $-1.4<[\mathrm{M} / \mathrm{H}]<-0.9$ and younger ages for high-metallicity clusters. This discrepancy is in part due to the different methods applied to measure the observed cluster parameters (see discussion in De Angeli et al. 2005, and in particular their Figure 7), and in part to the different evolutionary models adopted for the age determination (De Angeli et al. 2005, uses the P04 models, which we have shown to give consistent results with our adopted D07 models).

\subsection{The Special Case of NGC 0288 and NGC 0362}

In this study MSTO's magnitude is measured for each GGCs in our database, and then obtained magnitudes are transformed into ages using a set of theoretical stellar evolution models. During this transformation, canonical chemical abundances are assumed for all GGCs. We are aware of the fact that if a particular cluster has different chemical abundances, for example, an anomalous $\mathrm{CNO}$ content, then its age determination could be affected by this effect. It is worth mentioning that age uncertainties could be underestimated in this study because of the uncertainties coming from the chemical inhomogeneities which, to date, are impossible to quantify.

In particular, our results indicate that NGC 0288 and NGC 0362 have the same age within $\pm 0.9 \mathrm{Gyr}$, while previous works (Sarajedini \& Demarque 1990; Bellazzini at al. 2001) have found NGC 0288 to be $1-2$ Gyr older than NGC 0362 . The relative ages from these studies come from a comparison of all the evolutionary sequences (MS, SGB, HB, etc.), and not on a measurement of the absolute magnitude of the MSTO, as in the

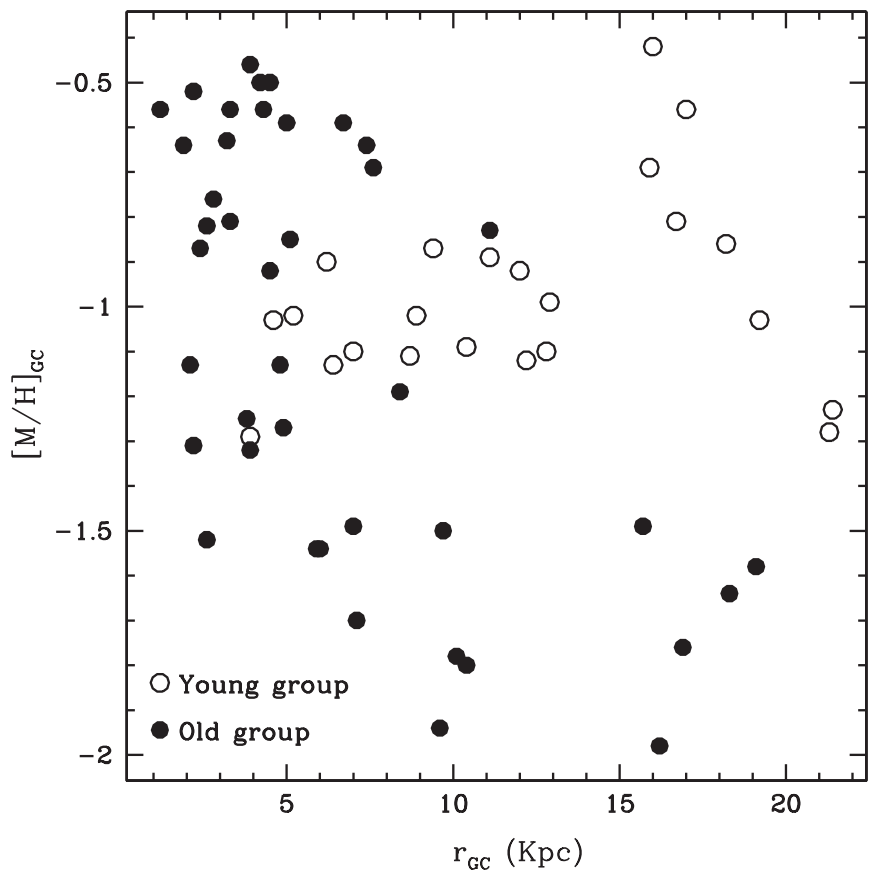

Figure 12. Globular cluster's $[\mathrm{M} / \mathrm{H}]$ in the CG-metallicity scale as a function of the galactocentric distance. Open and filled circles represent clusters in the young and old groups, respectively.

present paper. Therefore, we are planning a forthcoming paper in which we will, among other things, consider the case of these two clusters in more detail. In this context, it is worth mentioning that the present study does not take into consideration the distribution of stars along the $\mathrm{HB}$, or finer features such as the RGB tip luminosity, the RGB bump, AGB bump, and multiple populations.

A similar argument could be used for the case of NGC 5272 and NGC 6205, for which we obtain identical age, while 
previous works (VandenBerg et al. 1990; Chaboyer et al. 1996) have found NGC 6205 to be 1-2 Gyr older than NGC 5272.

\section{DISCUSSION}

Figure 10 shows the GGC normalized ages derived using the D07 stellar evolution library as a function of $[\mathrm{M} / \mathrm{H}]$ in the CG-metallicity scale (upper panel), and as a function of the galactocentric distance $\left(r_{\mathrm{GC}}\right.$, lower panel). The low-, intermediate-, and high-metallicity subgroups are separated by long dashed lines, and clusters belonging to the different metallicity groups are plotted with open circles (low-), filled triangles (intermediate-), and filled circles (high metallicity). The mean and dispersion of the low-metallicity clusters' age are marked with solid and dashed lines, respectively. For each of the three metallicity groups, mean age and $\mathrm{rms}$ are indicated. Figure 11 shows the same data as Figure 10, but for the ZWmetallicity scale.

Overall, we notice an increase in age dispersion (which is model independent) with the metallicity. However, we can look at the results shown in the upper panels of Figures 10 and 11 in another way. They show two branches in the age-metallicity relation of GGCs. On one hand, a "young" branch showing a clear age-metallicity relation, age decreasing at higher metallicities. On the other hand, a largely coeval "old" branch. The "old" branch shows an apparent relative age dispersion of $\sim 0.05$ and no age-metallicity relation. It is worth mentioning that while the age-metallicity relation may be model dependent (though it is somehow reassuring that the four most recent theoretical libraries provide consistent results on this respect) the age dispersion-metallicity relation is not model dependent. From now on, we will divide the clusters in two groups that we call "young" and "old". GGCs with total metallicity higher than -1.4 and normalized age younger than 0.95 , that is $1 \sigma$ smaller than the mean of the low-metallicity clusters' age, are considered members of the "young" group. The remaining GGCs are considered members of the "old" group. The only exception is NGC 6441, a very high-metallicity cluster which is much closer to the old branch than the young one, so it is included in the old group. Column 10 in Table 4 lists whether the target cluster belongs to the old or young group.

An age trend with a galactocentric distance is seen in the lower panels of Figures 10 and 11. The fraction of young group's clusters increases significantly as the galactocentric distance increases. As a result, age dispersion increases at the increasing galactocentric distance. Other authors have commented on the increased dispersion in ages at larger galactocentric distances. (e.g., Richer et al. 1996, which is one of the first to state the empirical problem clearly). Also in these panels, a clear metallicity trend with the galactocentric distance can be observed for the old group of clusters, with an increasing metallicity at lower galactocentric distances. This trend is explicitly shown in Figure 12, which plots GGC's [M/H] in the CG-metallicity scale as a function of the galactocentric distance. Open and filled circles represent clusters in the young and old groups, respectively.

In the following, the age dispersion of the old clusters group, the age-metallicity relation of the young clusters group and the age trend with galactocentric distance are discussed in detail.

\subsection{The Age Dispersion of the Old Clusters Group}

Figures 10 and 11 indicate that the mean normalized age of the clusters in the old group is the same for all metallicities. The relative age dispersion of this group is $\sim 0.05$. If an absolute age of $12.8 \mathrm{Gyr}$ (from the D07 models) is assumed as the mean age of these clusters, age dispersion would be $\sim 0.6$ Gyr. We note that if we assume that all sources of uncertainty have been taken into account in the error bars, then at least part of this dispersion must be real. The average normalized age uncertainty among the clusters in the old group is 0.04 , which is approximately $0.5 \mathrm{Gyr}$. Subtracting this quadratically from the observed dispersion, we find an intrinsic dispersion of 0.03 in relative age for the clusters in the old group. Using $12.8 \mathrm{Gyr}$ for the age of this group, the intrinsic dispersion is $\sim 0.4 \mathrm{Gyr}$.

These results are consistent with a scenario in which the old group of clusters formed within a fast assembling process of the halo, lasting $\sim 0.8 \mathrm{Gyr}$ and which should also account for an increasing metallicity toward the center of the Galaxy. This implies that the chemical enrichment of the protogalactic cloud was faster than the assembling timescale. Regarding the nature of such assembling process, it is interesting to note the following. Wilkinson \& Evans (1999) estimate the mass and scale length of the Milky Way dark matter halo to be $\sim 1.9 \times 10^{12} M_{\odot}$ and $170 \mathrm{kpc}$, respectively. The free-fall time of a homogeneous sphere of those mass and radius is $\sim 0.84 \mathrm{Gyr}$, similar to the age range we find here for the old clusters. In other words, the age dispersion of old globular clusters is not in contradiction with the formation from the colapse of a single protosystem, resembling the model proposed by Eggen et al. (1962). On the other side, the standard $\Lambda \mathrm{CDM}$ scenario would predict a general star formation, including star clusters, in protogalaxy building blocks of mass $\sim 10^{8} M_{\odot}$ before re-ionization (Moore et al. 2006). These blocks merge together afterward to form larger galaxies, and would, in principle, be compatible with the old, coeval cluster population that we observe. More difficult seems to account for the metallicity gradient (Figure 12) under this scenario. A more detailed analysis is necessary, but it is important to note that any successful galaxy formation scenario must account for the existence of a large nubmer of old, coeval globular clusters in the Milky Way.

\subsection{The Age-Metallicity Relation of the Young Clusters Group}

Clusters in the young group, however, show a clear agemetallicity relationship, with younger clusters being more metal rich than older ones. The nature of this "young branch" can perhaps be better understood by looking at the GGCs associated with known or putative accreted dwarf galaxies: Sagittarius, Canis Major, and Monoceros.

Ibata et al. (1995) discovered the accreted Sagittarius dwarf galaxy and its GC system (Terzan 7, Terzan 8, Arp 2, and M54). Dinescu et al. (2000) and Bellazzini et al. (2003) have since then argued, respectively, that Pal 12 and NGC 4147 are part of the extended tidal stream.

The Monoceros Ring was discovered by Newberg et al. (2002), and it has been proposed to be the tidal stream of the accreted Monoceros galaxy. Crane et al. (2003) and Frinchaboy et al. (2004) identified five possible GC candidates of this accreted galaxy: NGC 2298, NGC 2808, NGC 5286, Pal 1, and BH 176. In addition, Martin et al. (2004) identify four GGCs-NGC 1851, NGC 1904, NGC 2298, and NGC 2808possibly associated with a stellar overdensity in Canis Major, which they suspect to be a dwarf galaxy. However, the nature of these two structures is controversial. The existence of the Canis Major dwarf galaxy has been seriously questioned by Momany et al. (2004, 2006). In Momany et al. (2006), the Canis Major stellar overdensity has been completely accounted for as the 

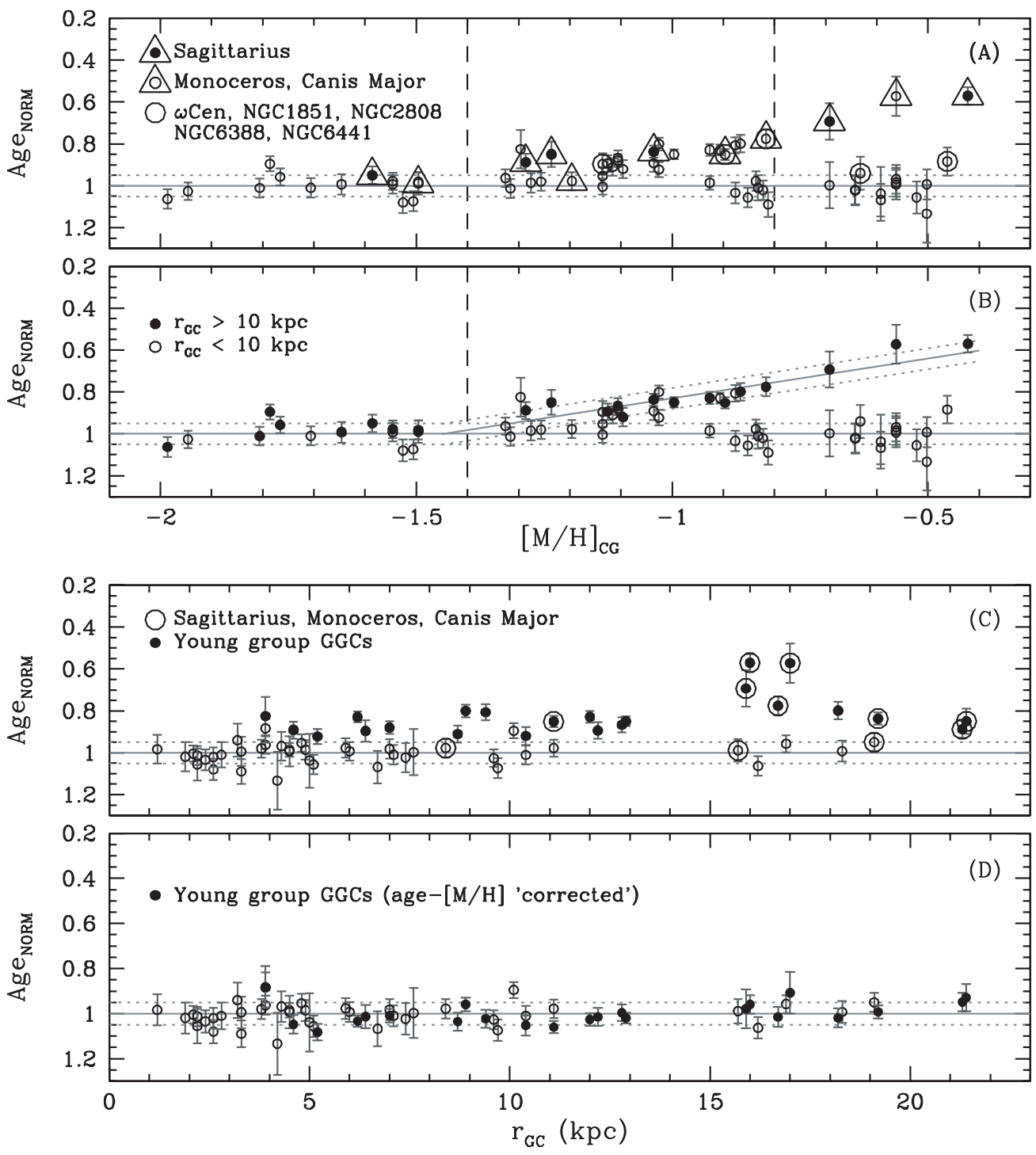

Figure 13. Age-metallicity relation using the CG-metallicity scale ( $a$ and $b)$ and normalized ages vs. galactocentric distance $(c$ and $d$ ). In $a$, clusters associated with Sagittarius, Monoceros, and Canis Major have been marked, together with multiple stellar population clusters. In $b$, GGCs with $r_{\mathrm{GC}}<10 \mathrm{kpc}$ are represented with open circles, while those with $r_{\mathrm{GC}}>10 \mathrm{kpc}$ are plotted with filled circles. A least-squares fit to the young group's age-metallicity relation is also plotted. In $c$, Sagittarius, Monoceros, and Canis Major' clusters are also marked. Finally, $d$ shows the same as $c$, but here ages of clusters in the young group have been age-metallicity "corrected" using the age-metallicity relation shown in $b$. See text for details.

effect of the Milky Way disk warp (an ubiquitous property of all massive galaxies). In addition, Momany et al. (2006) have shown that also the Monoceros Ring seems to reflect the signature of another Galactic disk property; the flaring (the increase in scale height as a function of Galactocentric distance) of the outer disk.

Figures 13(a) and (b) show the age-metallicity relation, using the CG-metallicity scale. ${ }^{14}$ Sagittarius, Monoceros, and Canis Major's clusters have been marked. It is interesting that, for metallicities higher than $[\mathrm{M} / \mathrm{H}]=-1.4$, all of Sagittarius, Monoceros, and Canis Major's accreted clusters fall in the young group, with the exception of NGC 5286, and they follow the same age-metallicity relation as the rest of the young group's clusters. This result suggests a different origin for the old and young groups of clusters. It is worth mentioning that there is a number of clusters, other than the Sagittarius, Monoceros, and Canis Major, clusters among the young sample.

\footnotetext{
14 Because the results are equivalent to those using the ZW-metallicity scale,
} we only consider the CG scale after this point.
Another interesting question is related to the recent discovery that GGCs are not simple single stellar populations. The high-mass and multiple stellar populations of $\omega$ Cen has led to speculation that it is the remnant nucleus of an accreted Milky Way satellite galaxy (Lee et al. 1999; Villanova et al. 2007). But $\omega$ Cen is not a unique case. NGC 2808 (Piotto et al. 2007), NGC 1851 (Milone et al. 2008), NGC 6388 (Piotto 2008), and NGC 6441 (Caloi \& D'Antona 2007) are four massive clusters hosting multiple stellar populations. Freeman (1993) suggested that GGCs form as the nuclei of dwarf galaxies in the early universe, and are accreted as their host galaxies merge onto larger structures. In this context, these peculiar GGCs would be good candidates to be the remnants of accreted satellites. Multiple population clusters have been also marked in Figure 13(a).

We note that multiple stellar population clusters have broadened MSTOs, and at least a fraction of the stars in these clusters have rather anomalous chemical compositions. For this reason, we should be hesitant in adopting their relative ages obtained in 
this study. A much more detailed analysis is needed for these peculiar clusters. Villanova et al. (2007) and Cassisi et al. (2008) present particularly illuminating discussions of the difficulties in estimating ages for the multiple populations in $\omega \mathrm{Cen}$ and NGC 1851, respectively.

Figure 13(b) shows the same as Figure 13(a), but here GGCs with $r_{\mathrm{GC}}>10 \mathrm{kpc}$ are represented with filled circles, while those with $r_{\mathrm{GC}}<10 \mathrm{kpc}$ are plotted with open circles. Interestingly, all intermediate- and high-metallicity clusters with $r_{\mathrm{GC}}>10$ $\mathrm{kpc}$ fall on the young group of clusters.

In order to characterize the young group's age-metallicity relation, a least-squares fit has been performed, and results are also shown in Figure 13(b). The age-metallicity relation observed in the young group of clusters can be described by the equation:

$$
\mathrm{Age}_{\mathrm{NORM}}=-0.38[\mathrm{M} / \mathrm{H}]+0.45 \text {. }
$$

This relation is plotted with a solid line in Figure 13(b). The dispersion of the clusters' relative ages with respect to the previous equation is also 0.05 , and it is also represented in Figure 13(b) (dashed lines). Following a similar argument as for the old group, if an absolute age of $12.8 \mathrm{Gyr}$ is assumed as the mean of the clusters in the old group, the young group's age dispersion with respect to the previous age-metallicity relation would be $\sim 0.6$ Gyr. The average normalized age uncertainty among the clusters in the young group is 0.04 , which is approximately 0.5 Gyr. Subtracting this quadratically from the observed dispersion, we find an intrinsic dispersion with respect to its age-metallicity relation of 0.03 in relative age, or $\sim 0.4$ Gyr.

In summary, our results are consistent with the clusters of the young group having been formed in a different process spanning an interval of time as long as 0.45 in relative age, or $\sim 6 \mathrm{Gyr}$, and resulting in a group of GCs with a clear age-metallicity relation. It is very tempting to argue that the origin of this second group of clusters is related to their formation within Milky Way satellite galaxies that were later accreted. However, the reason why the age-metallicity relation of the GGCs associated with the Sagittarius dwarf galaxy, as well as Monoceros and Canis Major, is similar to that of the rest of "accreted" clusters should be investigated. Perhaps all of the young group clusters share the same origin, which would require an association between Sagittarius, Monoceros, and Canis Major, or alternatively, all dwarf galaxy systems may share a common (or very similar) star formation and metal enrichment history, and hence a common age-metallicity relation, which seems unlikely.

\subsection{Age Trend with Galactocentric Distance}

With the aim of analyzing in more detail the observed age trend with galactocentric distance, GGCs in the old and young groups are considered separately. Figure 13(c) shows normalized ages versus galactocentric distance (same as Figure 10, lower panel), but now clusters in the old group are represented with open circles and clusters in the young group with filled circles. Besides, Sagittarius, Monoceros, and Canis Major's clusters are shown as encircled points.

It can be seen that if the old group is considered, an age trend with the galactocentric distance is not observed. The age's variance remains constant with the galactocentric distance for this group.

On the other hand, if the young group is taken into account, it can be seen that the age's variance increases with the galactocentric distance. In order to determine if this variance

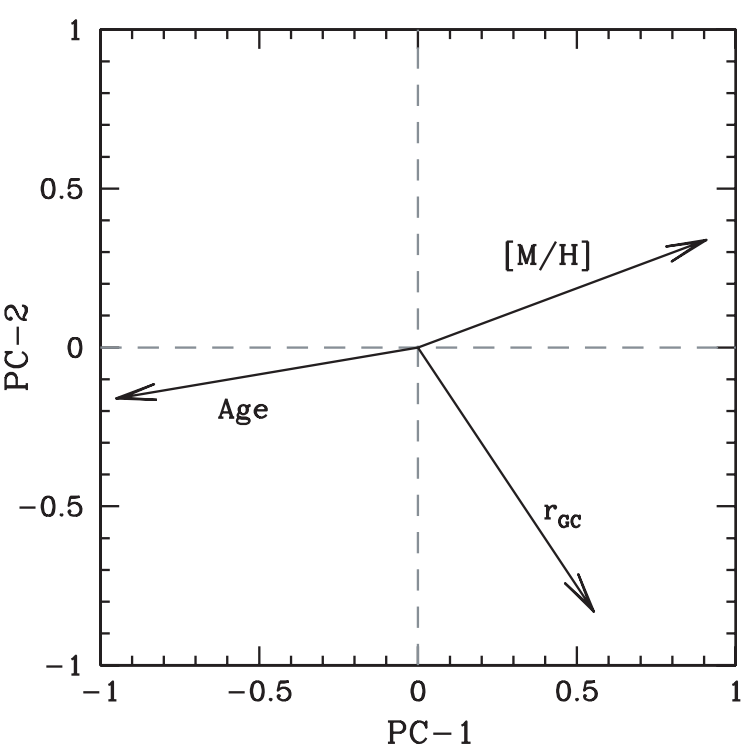

Figure 14. Young group principal-component (PC) analysis: relations between original data and PCs on the first two PCs plane. It can be seen that the first $\mathrm{PC}$ is strongly correlated with $[\mathrm{M} / \mathrm{H}]$ and age, but weakly with $r_{\mathrm{GC}}$, while the second $\mathrm{PC}$ carries on most of the $r_{\mathrm{GC}}$ variance. See text for details.

increase depends either on the galactocentric distance or on the metallicity, a three-dimensional principal-component (PC) analysis has been done considering the clusters in the young group only. The obtained eigenvalues are $2.03,0.83$, and 0.14 . The first PC, of eigenvalue 2.03, accounts for $68 \%$ of the total variance. According to the commonly used average or eigenvalue-one criterion, only this $\mathrm{PC}$ should be retained. Figure 14 shows the relations between the original data and PCs projected on the two first PCs plane. On the one hand, it can be seen that the first $\mathrm{PC}$ is strongly correlated with $[\mathrm{M} / \mathrm{H}]$ and age, but weakly with $r_{\mathrm{GC}}$, while the second PC carries on most of the $r_{\mathrm{GC}}$ variance. On the other hand, it is clear that young cluster age dispersion can be explained by an age-metallicity relation, and that age is not related to $r_{\mathrm{GC}}$.

In fact, the effect of metallicity on age of clusters in the young group can be eliminated by subtracting the fitted age-metallicity relation (Equation (4)) to the cluster's ages. By doing this, agemetallicity "corrected" ages are obtained. Figure 13(d) shows the same as Figure 13(c), but here age-metallicity "corrected" clusters are represented with solid circles. It can be clearly seen that most of the age dispersion observed in Figure 13(c) disappears, and that age is not related to $r_{\mathrm{GC}}$

In summary, it can be concluded that a significant part of the age dispersion present in Figure 13(c) for $r_{\mathrm{GC}} \leqslant 15 \mathrm{kpc}$ and most of that for $r_{\mathrm{GC}}>15$ can be explained by a strong age-metallicity relation for the clusters of the young group.

It is worth mentioning that our database is biased in distance. No outer halo clusters, with galactocentric distances larger than $\sim 20 \mathrm{kpc}$, have been considered for this study. So the analysis of the galactic halo's age structure is limited by the galactocentric distance range of the database. Given that outer-halo clusters (Pal 3, Pal 4, Pal 14, Eridanus, AM-1) have younger ages (Sarajedini 1997; Stetson et al. 1999; Dotter et al. 2008), it is possible that our age range might be a lower limit on the actual range.

\section{CONCLUSIONS}

Normalized ages have been derived for a sample of 64 GGCs using the stellar evolution models of D07 and both ZW- and 
CG-metallicity scales. We have also performed an analysis using the stellar models of P04, B94, and G00. The result is the most extensive and precise database of normalized ages so far produced. Our results are are as follows.

1. We find that we are able to measure relative ages to a formal precision of $2 \%-7 \%$. The relative ages are independent of the choice of the theoretical model. Four independent sets of stellar evolution libraries (D07, P04, B94, G00) produce essentially identical results.

2. We find that the GGCs fall into two well-defined groups. The first one represents a population of old clusters that have the same age, a dispersion of 5\% in relative age and no age-metallicity relation. If we assume an absolute age of $12.8 \mathrm{Gyr}$, the absolute age dispersion of the old clusters is $\sim 0.6$ Gyr. Accounting for the measurement uncertainties, we obtain an intrinsic age dispersion of $\sim 0.4 \mathrm{Gyr}$. The second group of clusters shows a clear age-metallicity relation, with young clusters being more metal rich than older ones. Also in this case, the intrinsic age dispersion with respect to its age-metallicity relation is $\sim 0.4 \mathrm{Gyr}$. Besides, there is no age dispersion-metallicity relation if we look at the two samples (old and young) separately.

3. These results are consistent with a scenario in which the formation of the Milky Way CCG system took place in two phases. The first one produced clusters with ages within a range of $\sim 0.8 \mathrm{Gyr}$. This age range is compatible with the timescale for the collapse of a protogalaxy of the same mass and scale length as that of the Milky Way dark matter halo. In other words, the age dispersion of the old group of globular clusters is not in contradiction with the formation from the collapse of a single protosystem, resembling the model proposed by Eggen et al. (1962). The standard $\Lambda \mathrm{CDM}$ scenario would also predict a significant star formation in protogalactic building blocks before reionization, which, after merging, would produce an old, coeval population of globular clusters in a large galaxy like the Milky Way. However, to account for the galactocentric metallicity gradient of this group seems difficult in the context of this mechanism. The second phase spanned a time interval as long as $\sim 6 \mathrm{Gyr}$ and resulted in a group of GGCs with a clear age-metallicity relation. It is very tempting to argue that the origin of this second group of clusters is related to their formation within Milky Way satellite galaxies that were later accreted. However, this would not account for the fact that the clusters of this group share the same age-metallicity relation.

4. We find that the increasing dispersion in age with the galactocentric distance is explained by the age-metallicity relation of GGCs.

5. These results are independent of the assumed metallicity scale.

6. It is worth mentioning that our database is biased by distance. No outer halo clusters, with galactocentric distances larger than $\sim 20 \mathrm{kpc}$, have been considered. The analysis of the galactic halo's age structure is therefore limited by the galactocentric distance range of the database.

Support for this work (proposal GO-10775) was provided by NASA through a grant from the Space Telescope Science Institute, which is operated by the Association of Universities for Research in Astronomy, Inc., under NASA contract NAS526555. A.M.F. has been partially supported by the Gran Telescopio Canarias Postdoctoral Fellowhip through the University of Florida and the Education and Research Ministry of Spain's "Juan de la Cierva" postdoctoral position. This work has been financially supported by the Instituto de Astrofísica de Canarias (grant P3-94) and the Education and Research Ministry of Spain (grant PNAYA2004-06343). G.P. and A.P.M. acknowledge partial support by the Agenzia Spaziale Italiana.

\section{REFERENCES}

Anderson, J., et al. 2008, AJ, 135, 2055

Aparicio, A., \& Gallart, C. 2004, AJ, 128, 1465

Arp, H. C., Baum, W. A., \& Sandage, A. R. 1953, AJ, 58, 4

Baade, W. 1944, ApJ, 100, 137

Bedin, L. R., Piotto, G., Anderson, J., Cassisi, S., Momany, Y., \& Carraro, G. 2004, ApJ, 605, L125

Bellazzini, M., Ferraro, F. R., \& Ibata, R. 2003, AJ, 125, 188

Bellazzini, M, Pecci, F. F., Ferraro, F. R., Galleti, S., Catelan, M., \& Landsman, W. B. 2001, AJ, 122, 2569

Bertelli, G., Bressan, A., Chiosi, C., Fagotto, F., \& Nasi, E. 1994, A\&AS, 106 275

Buonanno, R., Corsi, C. E., Pulone, L., Fusi Pecci, F., \& Bellazzini, M. 1998, A\&A, 333, 505

Caloi, V., \& D'Antona, F. 2007, A\&A, 463, 949

Carney, B. W. 1996, PASP, 108, 900

Carretta, E., Cohen, J. G., Gratton, R. G., \& Behr, B. B. 2001, AJ, 122, 1469

Carretta, E., \& Gratton, R. G. 1997, A\&AS, 121, 95

Cassisi, S., Salaris, M., Pietrinferni, A., Piotto, G., Milone, A. P., Bedin, L. R., \& Anderson, J. 2008, ApJ, 672, L115

Chaboyer, B., Demarque, P., Kernan, P. J., \& Krauss, L. M. 1998, ApJ, 494, 96

Chaboyer, B., Demarque, P., \& Sarajedini, A. 1996, ApJ, 459, 558

Crane, J. D., Majewski, S. R., Rocha-Pinto, H. J., Frinchaboy, P. M., Skrutskie, M. F., \& Law, D. R. 2003, ApJ, 594, L119

De Angeli, F., Piotto, G., Cassisi, S., Busso, G., Recio-Blanco, A., Salaris, M., Aparicio, A., \& Rosenberg, A. 2005, AJ, 130, 116

Dinescu, D. I., Majewski, S. R., Girard, T. M., \& Cudworth, K. M. 2000, AJ, 120,1892

Dotter, A., Chaboyer, B., Jevremović, D., Baron, E., Ferguson, J. W., Sarajedini, A., \& Anderson, J. 2007, AJ, 134, 376

Dotter, A., Sarajedini, A., \& Yang, S. C. 2008, AJ, 136, 1407

Eggen, O. J., Lynden Bell, D., \& Sandage, A. R. 1962, ApJ, 136, 748

Freeman, K. C. 1993, ASPC, 48, 608

Frinchaboy, P., Majewski, S. R., Crane, J. D., Reid, I. N., Rocha-Pinto, H. J., Phelps, R. L., Patterson, R. J., \& Munoz, R. R. 2004, ApJ, 602, L21

Girardi, L., Bressan, A., Bertelli, G., \& Chiosi, C. 2000, A\&AS, 141, 371

Gratton, R. G. 1985, A\&A, 147, 169

Gratton, R. G., Bragaglia, A., Carretta., Clementini, G., Desidera, S., Grundahl, F., \& Lucatello, S. 2003, A\&A, 408, 529

Harris, W. E. 1996, AJ, 112, 1487

Ibata, R., Gilmore, G., \& Irwin, M. 1995, MNRAS, 277, 781

Kirby, E. N., Guhathakurta, P., \& Sneden, C. 2008, ApJ, 682, 1217

Lee, Y. W., Joo, J. M., Sohn, Y. J., Rey, S. C., Lee, H. C., \& Walker, A. R. 1999, Nature, 402, 55

Martin, N., Ibata, R., Bellazzini, M., Irwin, M., Lewis, G., \& Dehnen, W. 2004, MNRAS, 348, 12

Meissner, F., \& Weiss, A. 2006, A\&A, 456, 1085

Milone, A. P., et al. 2008, ApJ, 673, 241

Momany, Y., Zaggia, S. R., Bonifacio, P., Piotto, G., De Angeli, F., Bedin, L. R., \& Carraro, G. F. 2004, A\&AS, 421, 29

Momany, Y., Zaggia, S., Gilmore, G., Piotto, G., Carraro, G., Bedin, L. R., \& de Angeli, F. 2006, A\&AS, 451, 515

Moore, B., Diemand, J., Madau, P., Zemp, M., \& Stadel, J. 2006, MNRAS, 368, 563

Newberg, H., et al. 2002, ApJ, 569, 245

Oort, J. H. 1926, The Observatory, 49, 302

Pancino, E., Ferraro, F. R., Bellazzini, M., Piotto, G., \& Zoccali, M. 2000, ApJ, 534, L83

Peterson, C. J. 1987, PASP, 99, 1153

Pietrinferni, A., Cassisi, S., Salaris, M., \& Castelli, F. 2004, ApJ, 612, 168

Piotto, G. 2008, in XXI Century Challenges for Stellar Evolution, ed. S. Cassisi \& M., MemSAIt Salaris, 79, 334

Piotto, G., et al. 2002, A\&A, 391, 945

Piotto, G., et al. 2007, ApJ, 661L, 53

Richer, H. B., et al. 1996, ApJ, 463, 602

Rosenberg, A., Aparicio, A., Saviane, I., \& Piotto, G. 2000b, A\&AS, 145, 45

Rosenberg, A., Saviane, I., Piotto, G., \& Aparicio, A. 1999, AJ, 118, 2306 
Rosenberg, A., Piotto, G., Saviane, I., Aparicio, A., \& Gratton, R. 1998, AJ, 115,648

Rosenberg, A., Piotto, G., Saviane, I., \& Aparicio, A. 2000a, A\&AS, 144, 5

Rutledge, G. A., Hesser, J. E., \& Stetson, P. B. 1997, PASP, 109, 907

Salaris, M., \& Cassisi, S. 1996, A\&A, 305, 858

Salaris, M., Chieffi, A., \& Straniero, O. 1993, ApJ, 414, 580

Salaris, M., \& Weiss, A. 1998, A\&A, 335, 943

Sandage, A. R. 1953, AJ, 58, 61

Sarajedini, A., \& King, C. R. 1989, AJ, 98, 1624

Sarajedini, A., \& Demarque, P. 1990, ApJ, 65, 219

Sarajedini, A. 1997, AJ, 113, 682

Sarajedini, A., Chaboyer, B., \& Demarque, P. 1997, PASP, 109, 132
Sarajedini, A., et al. 2007, AJ, 133, 1658

Siegel, M. H., et al. 2007, ApJ, 667L, 57

Stetson, P. B., Vandenberg, D. A., \& Bolte, M. 1996, PASP, 108, 560

Stetson, P. B., et al. 1999, AJ, 117, 247

Tavarez, M., \& Friel, E. D. 1995, AJ, 110, 223

VandenBerg, D. A. 2000, ApJS, 129, 315

VandenBerg, D. A., Bolte, M., \& Stetson, P. B. 1990, AJ, 100, 445

Venn, K. A., Irwin, M., Shetrone, M. D., Tout, C. A., Hill, V., \& Tolstoy, E 2004, AJ, 128, 1177

Villanova, et al. 2007, ApJ, 663, 296

Wilkinson, M. I., \& Evans, N. W. 1999, MNRAS, 310, 645

Zinn, R., \& West, M. J. 1984, ApJS, 55, 45 